\title{
Zulässigkeit und Grenzen mitgliedstaatlicher Eingriffe in die freie Preisgestaltung bei Agrarerzeugnissen Rechtsdogmatische Überlegungen im Lichte der Rechtsprechung der Europäischen Gerichtshofes
}

\section{Preisregelungen als Instrument der Gemeinsamen Agrarpolitik}

In welchem Umfang die Mitgliedstaaten in die freie Preisgestaltung bei Agrarerzeugnissen eingreifen dürfen, ist eine grundlegende Frage des EU-Agrarmarktrechts und passt daher gut zu den intensiven agrarrechtsdogmatischen Forschungen, die der mit dem vorliegenden Beitrag geehrte Prof. Dr. Roman Budzinowski unternommen hat. Als das EU-Agrarmarktrecht 1962 begann, besaßen die damaligen sechs Mitgliedstaaten jeweils eine eigene Agrarmarktordnung ${ }^{1}$. Diese nationalen Agrarmarktordnungen mit ihren Binneninstrumenten und Außenschutzmechanismen wirkten primär preissteuernd. Für die wichtigsten Agrarerzeugnisse war ein bestimmter Binnenmarktpreis gewollt, der durch Außenhandelsmechanismen wie Einfuhrzölle und Ausfuhr-

* Friedrich-Wilhelms-Universität Bonn, Deutschland. Vorliegend geäußerte Ansichten sind rein persönlicher Natur.

${ }^{1}$ Vgl. zur Vorgeschichte und Entwicklung des EU-Agrarmarktrechts von 1958 bis 2013 C. Busse, Zu Entstehung und Inhalt der Verordnung (EG) Nr. 1234/2007 über eine gemeinsame Organisation der Agrarmärkte (Einheitliche GMO), ,Jahrbuch des Agrarrechts“ 2008, Band VIII, Nr. 3, mit weiteren Nachweisen. 
kontrollen abgesichert wurde. Um einen EU-Agrarbinnenmarkt zu verwirklichen, mussten die nationalen Agrarmarktordnungen abgebaut werden. Denn ein funktionierender Binnenmarkt bedingt, dass entweder ein freier Preiswettbewerb herrscht oder einheitliche Preisregelungen für alle Marktteilnehmer bestehen. Folglich sah Art. 43 Abs. 1 Unterabs. 1 AEUV „die Ablösung der einzelstaatlichen Marktordnungen“ durch ,gemeinsame Organisationsformen“ vor. Diese ,gemeinsamen Organisationsformen“ bestimmte Art. 40 Abs. 1 Unterabs. 2 AEUV näher. Nach Art. 40 Abs. 1 Unterabs. 1 AEUV bildeten die „gemeinsamen Organisationsformen" für die einzelnen Erzeugnisbereiche zusammen genommen die ,gemeinsame Organisation der Agrarmärkte“. Sämtliche dieser Bestimmungen des AEUV existieren auch heute noch, so dass der AEUV nach wie vor eine gemeinsame Organisation der Agrarmärkte statuiert.

Art. 40 Abs. 2 Unterabs. 1 und 3 sowie Art. 43 Abs. 3 AEUV erwähnen als ein Instrument der gemeinsamen Organisation der Agrarmärkte „Preisregelungen“, eine „gemeinsame Preispolitik“ und die „Festsetzung der Preise“. Besonders betont wird zudem, dass die Preispolitik ,,auf gemeinsamen Grundsätzen und einheitlichen Berechnungsmethoden" beruhen muss. Das Instrument der Preisregelung wurde intensiv genutzt, als die EU ihre Agrarmarktorganisation errichtete. Während einer Übergangsphase bis 1970 fanden sich für die wichtigsten Agrarerzeugnisse Referenzpreise auf EU-Ebene festgelegt. Die zugehörigen Interventionsmechanismen - vor allem die öffentliche Lagerhaltung und die Beihilfe für die private Lagerhaltung - und der Außenschutz waren so ausgelegt, dass durch sie das Referenzpreisniveau in der EU erreicht wurde.

Damit dieses System funktionieren konnte, mussten die Mitgliedstaaten ihre nationalen Preissysteme aufheben. Mit dem Ende der Übergangsphase 1970 war der EU-Agrarbinnenmarkt weitgehend vollendet. Die Mitgliedstaaten durften ab jetzt keine neuen Preisregelungen mehr schaffen, wie die wohl allgemeine Ansicht annahm. Mit der MacSharry-Reform 1992 begann die EU, die unmittelbar preisbeeinflussenden Interventionsmechanismen teilweise abzubauen. An ihre Stelle traten gekoppelte Produktionsbeihilfen, die mittelbar preisbestimmend waren, so dass zusammen mit den verbliebenen Interventionsmechanismen nach wie vor ein EU-Preisregime bestand.

Erst mit der Entkopplung der Beihilfen von der Produktion bestimmter Agrarerzeugnisse und der weiteren Rückführung der Interventionsmechanismen kam das EU-Preisregime zu einem grundsätzlichen Ende. Das System der Referenzpreise fiel weg. Stattdessen wurde ein so genanntes Sicherheitsnetz etabliert, das für mehrere wichtige Agrarerzeugnisse bei einem starken Absinken der EU-Binnenmarktpreise eingreift. Zu diesem Zweck wurden Referenzschwellenwerte definiert, bei deren Unterschreitung die Interventionsmechanismen zur Anwendung kommen. Zugleich fand sich das 
bestehende Kriseninstrumentarium ausgebaut, so dass während erheblicher Marktkrisen stützende Eingriffe in den Markt möglich sind. Parallel dazu senkte die EU ihren Außenschutz.

Im Ergebnis bilden sich die Preise für Agrarerzeugnisse nun im Wesentlichen am Markt. Durch den nur noch geringen Außenschutz werden diese Marktpreise in hohem Maße von dem Preisniveau auf dem Weltagrarmarkt beeinflusst. Zwar wirkt auch das entkoppelte Beihilfensystem auf das Preisniveau. Diese Wirkung ist jedoch von generell preisdämpfender Natur und schlägt sich regelmäßig nicht in direkten Preiseffekten für einzelne Erzeugnisse nieder. Anders verhält es sich mit den wenigen nach wie vor bestehenden gekoppelten Beihilfen und teilweise auch mit den so genannten Sektorprogrammen, die jeweils für einzelne Agrarerzeugnisbereiche bestehen und in unterschiedlichem Maße von den Mitgliedstaaten genutzt werden. Insbesondere die gekoppelten Beihilfen besitzen dabei das Potential, den Wettbewerb zwischen den Mitgliedstaaten zu verzerren. Erwähnt sei etwa die gekoppelte Beihilfe im Zuckerbereich, die in Polen und einigen anderen Mitgliedstaaten gewährt wird, in Deutschland hingegen nicht besteht.

Ursprünglich war zur Vermeidung von Wettbewerbsverzerrungen festgelegt, dass eine gekoppelte Beihilfe - so Art. 52 Abs. 5 VO (EU) Nr. 1307/2013 in seiner Erstfassung - nur gewährt werden durfte, „um einen Anreiz zur Beibehaltung des derzeitigen Produktionsniveaus in den betreffenden Sektoren oder Regionen zu schaffen“. Da eine gekoppelte Beihilfe jedoch regelmäßig automatisch einen Anreiz für eine Ausdehnung des Produktionsniveaus setzt, hätte die Europäische Kommission eigentlich die meisten der ihr von den Mitgliedstaaten notifizierten gekoppelten Beihilfen nicht billigen dürfen. Die Europäische Kommission setzte sich jedoch über das Kriterium hinweg, so dass es Anfang 2018 auf Druck derjenigen Mitgliedstaaten, die derartige gekoppelte Beihilfe gewähren, gestrichen wurde. Damit steht den Mitgliedstaaten ein Instrument zur Verfügung, mit dem sie - in geringem Umfang - indirekt preissteuernd tätig sein können. Dieses Instrument entstammt dabei allerdings nicht dem nationalen Recht, sondern dem EU-Recht.

\section{Die Auffassung vom impliziten Verbot nationaler Preisregelungen}

Soweit für den Verfasser ersichtlich ist, wurde die Frage, wie das allgemein angenommene Verbot nationaler Preisregelungen im Agrarmarktbereich rechtlich genau zu begründen ist, bislang noch nicht näher untersucht. Dies dürfte daran liegen, dass das Verbot jahrzehntelang völlig unstreitig war, da 
es faktisch zwingend aus der umfassenden EU-rechtlichen Regulierung des Agrarmarktes folgte.

Auffallend ist zunächst, dass das Verbot im EU-Agrarmarktrecht nicht explizit normiert ist. So fand sich weder in den früheren Einzelmarktordnungen noch in der aus ihnen hervorgegangenen Verordnung (EG) Nr. 1234/2007 eine solche horizontal wirkende Bestimmung, die jeweils gleich hätte formuliert sein können. Auch in dem jetzigen EU-Agrarmarktkodex in Gestalt der Verordnung (EU) Nr. 1308/2013 (GMO) ist sie nicht vorhanden. Das Verbot mitgliedstaatlicher Preisregelungen wird daher regelmäßig implizit abgeleitet. Dem liegt die Annahme zu Grunde, dass die EU den Agrarmarkt umfassend und zugleich abschließend regeln möchte ${ }^{2}$. Folglich soll den Mitgliedstaaten nur noch dann nationales Agrarmarktrecht gestattet sein, wenn das EU-Agrarmarktrecht einen mitgliedstaatlichen Regelungsfreiraum vorsieht ${ }^{3}$. Von dieser Systematik werden insbesondere Preisregelungen erfasst. Zwar ordne - so geht die Argumentation weiter - Art. 4 Abs. 2 Buchstabe d AEUV die Landwirtschaft als Kompetenzbereich der EU in geteilter Zuständigkeit ein. Da die EU jedoch ihre Zuständigkeit im Agrarmarktbereich umfassend ausgeübt habe, könnten nach Art. 2 Abs. 2 Satz 2 AEUV die Mitgliedstaaten nicht mehr tätig werden ${ }^{4}$.

Je mehr die EU ihre agrarmarktsteuernden Regelungen abbaut, desto öfter wird allerdings eine solche Argumentation in Frage gestellt. Bestehen schlussendlich für einen Erzeugnisbereich keinerlei marktsteuernde Mechanismen mehr, so muss aus der Nichtexistenz solcher Regelungen abgeleitet werden, dass die EU den Mitgliedstaaten den Erlass derartiger Regelungen untersagt, ohne dass es dazu eine explizite Bestimmung im EU-Sekundärrecht zum Agrarmarkt gibt. Auch das EU-Primärrecht zum Agrarmarkt enthält in dieser Hinsicht keine explizite Aussage, da es auf den Aufbau einer gemeinsamen Agrarmarktorganisation und die damit notwendigerweise einhergehende Aufhebung der nationalen Agrarmarktordnungen ausgerichtet ist. Den entgegengesetzten Prozess des Abbaus der EU-Agrarmarktorganisation und damit

${ }^{2}$ Vgl. etwa P.P. Hase, Art. 38 AEUV, Rn. 13, in: M. Düsing, J. Martinez (Hrsg.), Agrarrecht, München 2016, S. 699 ff.: „Die sekundärrechtliche GMO entfaltet daher eine Sperrwirkung, was dazu führt, dass Mitgliedstaaten Maßnahmen nicht ermöglicht werden, wenn sie von der GMO abweichen oder deren Regeln verletzen“; ebenso R. Priebe, Art. 38 AEUV, Rn. 99, in: E. Grabitz, M. Hilf, M. Nettesheim (Hrsg.), Das Recht der Europäischen Union, 56. Ergänzungslieferung, München 2015: „'Sperrwirkung' der Marktordnungsregelung“.

3 Vgl. näher etwa C. Busse, Art. 43 AEUV, Rn. 15 ff., in: C.-O. Lenz, K.-D. Borchardt (Hrsg.), EU-Verträge-Kommentar, Köln 2013.

${ }^{4}$ Vgl. etwa T. van Rijn, Art. 38 AEUV, Rn. 1, in: H. von der Groeben, J. Schwarze, A. Hatje (Hrsg.), Europäisches Unionsrecht, Baden-Baden 2015: „Insoweit hat die Union eine exklusive Zuständigkeit.“ 
die Frage des Wiederauflebens mitgliedstaatlicher Agrarmarktkompetenzen hatten die Verfasser des EU-Primärrechts nicht vor Augen. Auch als der Abbauprozess bereits in vollem Gange war, wurde bei der ab 1992 erfolgten Neugestaltung des EU-Primärrechts durch die Verträge von Maastricht, Amsterdam, Nizza und Lissabon diese Frage nicht in Angriff genommen, sondern stattdessen das EU-Primärecht zum Agrarmarkt grundsätzlich unverändert beibehalten.

\section{Eine alternative Begründung des Verbots nationaler Preisregelungen}

Wird nach einer alternativen Begründung für das Verbot nationaler Preisregelungen gesucht, fällt der Blick auf die in Art. 40 Abs. 1 Unterabs. 2 AEUV erwähnten drei „Organisationsformen“. An der Spitze steht „eine Europäische Marktordnung“. Es folgen in absteigender Regelungsdichte die „bindende Koordinierung der verschiedenen einzelstaatlichen Marktordnungen" und „gemeinsame Wettbewerbsregeln“. Für fast alle Erzeugnisbereiche schuf die EU „europäische Markordnungen“. Die „bindende Koordinierung“ der nationalen Marktordnungen wurde nur in der Übergangsphase bis 1970 zur Angleichung unter anderem des Preisniveaus genutzt. Die Organisationsform der „gemeinsamen Wettbewerbsregeln“ kam soweit ersichtlich kaum zur Anwendung.

Allerdings gab es zu keiner Zeit für alle in Anhang I des AEUV genannten Agrarerzeugnisse eine preissteuernde Marktordnung. So wurde beispielsweise die EU-Bananenmarktordnung nach langer Diskussion und ausgelöst durch die Süderweiterung der EU erst 1993 geschaffen. Für Frühkartoffeln existierte nie eine Marktordnung, so dass die nationalen Marktordnungen für Frühkartoffeln - soweit solche wie etwa in Frankreich vorhanden waren - fortgeführt werden konnten. Theoretisch hätten auch neue nationale Marktordnungen mit direkten oder indirekten Preisregelungen für Frühkartoffeln eingeführt werden können. Dabei war allerdings zu berücksichtigen, dass Art. 38 Abs. 2 AEUV die allgemeinen Regelungen über den EU-Binnenmarkt für anwendbar erklärt, soweit nicht das Agrarkapitel des AEUV spezielle Regelungen enthält oder deren Erlass gestattet. Folglich durften auch im Frühkartoffelbereich keine direkten oder indirekten Handelshemmnisse im Sinne der Art. 34 AEUV ausdifferenzierenden Dassonville-Formel erlassen werden. Eine nationale Preisregelung im Frühkartoffelbereich hätte sich an dieser generellen Grenze für mitgliedstaatliche Maßnahmen messen lassen müssen. 
Erst als der einleitende Satz zu Teil XIV des Anhangs I der GMO ab 2014 pauschal alle zuvor nicht erfassten Anhang-I-Erzeugnisse zum Bestandteil der EU-Agrarmarktorganisation erklärte, wurden auch die Frühkartoffeln mit einbezogen. Die GMO kennt zwar nach wie vor keine nur auf Frühkartoffeln bezogenen Regelungen. Die horizontalen Instrumente der GMO wie beispielsweise das Agrarorganisationenrecht, die Kartellfreistellungen und die Krisenbestimmungen finden jedoch auf sie Anwendung, so dass seitdem nationale Marktordnungen zu Frühkartoffeln unanwendbar geworden sind. Diese grundsätzliche Konsequenz eines lediglich kurzen Vorspruchs zu dem letzten Teil eines der zahlreichen Anhänge zur GMO ist weitgehend unbemerkt geblieben.

Da nunmehr alle Anhang-I-Erzeugnisse ausdrücklich Bestandteil der GMO sind, dürfen keine nationalen Marktordnungen mehr existieren. Hiergegen lässt sich nicht einwenden, dass für eine Vielzahl von Erzeugnissen keine spezifisch auf das jeweilige Erzeugnis zugeschnittenen marktsteuernden Regelungen in der GMO existieren. Denn die Art des Instrumentes ist grundsätzlich für die Frage, ob eine gemeinsame Marktorganisation vorliegt, nicht relevant. So erlaubt Art. 40 Abs. 2 Unterabs. 1 AEUV zur "Gestaltung“ der EU-Agrarmarktorganisation ,alle zur Durchführung“ der Ziele des Art. 39 AEUV „erforderlichen Maßnahmen“. Die anschließende Aufzählung der klassischen marktsteuernden Instrumente ist nur beispielhaft und spiegelt den damaligen Stand des Agrarmarktrechts wider. Dass die EU inzwischen weitgehend auf direkt marktsteuernde Instrumente verzichtet hat und stattdessen auf Instrumente setzt, die den Marktbeteiligten helfen, den Markt selbst zu beeinflussen und sich damit an die neuen marktliberalen Gegebenheiten anzupassen, führt folglich nicht dazu, dass keine ,gemeinsame Organisation der Agrarmärkte" mehr vorliegt. Entsprechend lautet auch der Titel der GMO „Verordnung [...] über eine gemeinsame Marktorganisation für landwirtschaftliche Erzeugnisse".

Dieses Resultat wird auch nicht durch den generellen Rückbau der Agrarmarkteingriffe der EU in Frage gestellt. So wird zwar teilweise darauf hingewiesen, dass nach Art. 2 Abs. 2 Satz 3 AEUV die Zuständigkeit an die Mitgliedstaaten zurückfällt, ,,sofern und soweit die Union entschieden hat, ihre Zuständigkeit nicht mehr auszuüben“. Die EU übt jedoch ihre Zuständigkeit im EU-Agrarmarktbereich weiterhin umfassend aus, da wie dargestellt an die Stelle der Markteingriffsregelungen andere den Markt beeinflussende Instrumente der EU getreten sind.

Vor allem aber kann hinterfragt werden, ob überhaupt eine geteilte $\mathrm{Zu}-$ ständigkeit vorliegt. So findet sich in der Liste des Art. 3 AEUV mit den ausschließlichen Zuständigkeiten der EU in Abs. 1 Buchstabe b die „Festlegung 
der für das Funktionieren des Binnenmarktes erforderlichen Wettbewerbsregeln“. Art. 38 Abs. 1 Unterabs. 2 Satz 1 AEUV erklärt den Agrarmarkt zu einem Teil des EU-Binnenmarktes, auf den nach Art. 38 Abs. 2 AEUV die allgemeinen Regelungen des Binnenmarktes Anwendung finden, soweit nicht auf Grund des Landwirtschaftskapitels des AEUV Anderweitiges geregelt wird. Die EU-Agrarmarktorganisation legt die Regeln fest, in dessen Rahmen sich der Wettbewerb im Agrarbereich bewegt. Art. 40 Abs. 1 Unterabs. 2 Buchstabe a AEUV spricht sogar ausdrücklich von ,gemeinsamen Wettbewerbsregeln" als einer Organisationsform. Auch die beiden anderen in Art. 40 Abs. 1 Unterabs. 2 Buchstabe a AEUV genannten Organisationsformen beinhalten gemeinsame Wettbewerbsregeln, da die Koordinierung nationaler Marktordnungen und eine EU-Agrarmarktordnung die Regelung des Wettbewerbs inkludieren.

Art. 3 Abs. 1 Buchstabe b AEUV erläutert nicht näher, welche Bestimmungen des AEUV von ihm gemeint sind. Zumindest aber dürften diejenigen Bestimmungen gemeint sein, die im Zusammenhang mit dem EU-Binnenmarkt gemeinsame Wettbewerbsregeln aufstellen und in diesem Kontext sogar ausdrücklich von „Wettbewerbsregeln“ sprechen 5 . Folglich lässt sich vertreten, dass es sich bei der Zuständigkeit für die Errichtung einer EUAgrarmarktorganisation um eine ausschließliche Kompetenz handelt, während die landwirtschaftlichen Regelungen im Übrigen unter die geteilte Zuständigkeit nach Art. 4 Abs. 2 Buchstabe d AEUV fallen ${ }^{6}$. Dies hätte zur Folge, dass nicht Art. 2 Abs. 2 AEUV, sondern Art. 2 Abs. 1 AEV einschlägig ist. Die Mitgliedstaaten dürften mithin im Bereich der EU-Agrarmarktorganisation nur noch tätig werden, „wenn sie von der Union hierzu ermächtigt werden, oder um Rechtsakte der Union durchzuführen." Damit würde zugleich nach Art. 5 Abs. 3 EUV im Agrarmarktbereich das Subsidiaritätsprinzip nicht gelten. Dieses Ergebnis entspricht inhaltlich der landläufigen Ansicht, dass sich aus dem EU-Agrarmarktrecht implizit ergibt, dass die Mitgliedstaaten grundsätzlich kein eigenes Agrarmarktrecht mehr erlassen dürfen. Es verhindert jedoch zudem, dass im Falle eines teilweisen oder vollständigen Abbaus des

${ }^{5}$ Die Kommentarliteratur erörtert diesen Punkt regelmäßig nicht näher. So heißt es etwa bei W. Obwexer, Art. 3 AEUV, Rn. 13, in: H. von der Groeben, J. Schwarze, A. Hatje (Hrsg.), Europäisches Unionsrecht, lediglich recht vage: „Mit den Wettbewerbsregeln sind wohl die Bestimmungen in Teil III Titel VII Kap. 1 AEUV gemeint.“

${ }^{6}$ Ein Gegenargument ist, dass Art. 4 Abs. 2 Buchstabe d AEUV ausdrücklich die Rückausnahme des Art. 3 Abs. 1 Buchstabe d AEUV in Form der „Erhaltung der biologischen Meeresschätze im Rahmen der gemeinsamen Fischereipolitik“ nennt. Es existieren jedoch im Rahmen der Art. $101 \mathrm{ff}$. AEUV auch Sonderregelungen für mehrere in Art. 4 Abs. 2 AEUV aufgelistete Sachbereiche, ohne dass in Art. 4 Abs. 2 AEUV klargestellt wird, dass insofern die Kompetenzform des Art. 3 Abs. 1 Buchstabe b AEUV vorgeht. 
EU-Agrarmarktrechts die Kompetenz ohne eine ausdrückliche Anordnung im EU-Agrarmarktrecht an die Mitgliedstaaten zurückfallen könnte.

Unterstützt wird ein solches Resultat durch die Konzeption der Art. 40 und 43 AEUV, wonach an die Stelle der einzelstaatlichen Marktordnungen eine EU-Agrarmarktorganisation tritt. Ist dies geschehen, entsteht nicht eine bloß EU-sekundärrechtliche, sondern eine EU-primärrechtliche Sperrwirkung für nationales Agrarmarktrecht. Diese Sperrwirkung kann nur durch eine Ermächtigung im EU-Agrarmarktsekundärrecht durchbrochen werden, nationales Agrarmarktrecht zu setzen. Dass die Errichtung einer EU-Agrarmarktorganisation notwendig ist, hat bereits Art. 40 Abs. 1 Unterabs. 1 AEUV festgelegt, so dass für die Anwendung des Subsidiaritätsprinzips in Bezug auf die Frage der Errichtung kein Raum vorhanden ist. Die Grenzen des EU-rechtlichen Handelns normiert vielmehr Art. 40 Abs. 2 Unterabs. 1 und 2 AEUV selbst, indem er die zu treffenden Maßnahmen auf diejenigen begrenzt, die zur Erreichung der Ziele des Art. 39 AEUV „erforderlich“ sind, und zugleich davon spricht, dass sich die ,gemeinsame Organisation auf die Verfolgung der Ziele“ des Art. 39 AUV „zu beschränken“ hat.

Da sich eine Ermächtigung für die Mitgliedstaaten, wieder nationale Agrarmarktinstrumente einführen zu können, an den Zielen des Art. 39 AEUV für die gemeinsame Agrarpolitik ausrichten müsste, ist garantiert, dass keine rein nationalen Interessen verfolgbar werden können. Zu den bei einer derartigen Ermächtigung zu beachtenden Grundprinzipien gehört ebenfalls, dass das Funktionieren des EU-Agrarbinnenmarktes nicht gestört werden darf. Dass die GMO von diesen Grundgedanken durchzogen ist, zeigt sich etwa an Art. 210 Abs. 4 Buchstabe c GMO, wonach die Europäische Kommission Tätigkeiten von anerkannten Branchenverbänden nicht erlauben darf, die „Wettbewerbsverzerrungen hervorrufen können, die zur Erreichung der von der Branchenmaßnahme verfolgten Ziele der GAP nicht unbedingt erforderlich ist". Art. 149 Abs. 6 Unterabs. 1 und 152 Abs. 1c Unterabs. 1 GMO enthalten vergleichbare Gedanken. Vor allem aber erklären Art. 206 Unterabs. 1 und Art. 211 Abs. 1 GMO die allgemeinen Wettbewerbsregeln des AEUV für prinzipiell anwendbar, soweit die GMO keine anderweitigen Regelungen enthält.

\section{Die ständige Rechtsprechung des EuGH zu nationalen Agrarmarktregelungen}

Unabhängig von der genauen Begründung sind im Ergebnis nationale Preisregelungen im Bereich der Ziele des Art. 39 AEUV nur möglich, wenn im EU-Agrarmarktrecht eine entsprechende ausdrückliche oder implizi- 
te Ermächtigung für die Mitgliedstaaten vorhanden ist. Dies stimmt mit der langjährigen Rechtsprechung des Europäischen Gerichtshofes (EuGH) überein, die kurz skizziert werden soll. Eine der ersten grundlegenden Entscheidungen war das Urteil vom 23.1.1975 in der Rechtssache van der Hulst: „Wenn die [EU gemäß Art. 40 AEUV] eine Regelung über die Errichtung einer gemeinsamen Marktorganisation für einen bestimmten Sektor erlassen hat, sind die Mitgliedstaaten verpflichtet, sich aller Maßnahmen zu enthalten, die von dieser Regelung abweichen oder sie verletzen könnten"7. In der Rechtssache Prantl urteilte der EuGH am 13.3.1984: „Es ist richtig, dass die Mitgliedstaaten, sobald eine Regelung über die Errichtung einer gemeinsamen Marktorganisation als abschließend angesehen werden kann, in diesem Bereich keine Zuständigkeiten mehr haben, es sei denn, das [EU-Recht] sieht ausdrücklich etwas Anderes vor"

In dem Urteil des EuGH vom 25.3.2004, das in der Rechtssache Industrias de Deshidratación erging, hieß es: „Nach ständiger Rechtsprechung des Gerichtshofes sind die Mitgliedstaaten in dem von einer gemeinsamen Marktorganisation umfassten Bereich grundsätzlich nicht mehr befugt, durch einseitig erlassene innerstaatliche Rechtsvorschriften einzugreifen [...] Ihre Gesetzgebungszuständigkeit kann nur eine Restzuständigkeit sein und beschränkt sich auf die Situationen, die durch die [Unionsnorm] nicht geregelt sind, und die Fälle, in denen diese ihnen ausdrücklich Befugnisse einräumen [...]“. Weiter lautete es: „Ferner sind die Mitgliedstaaten, wenn eine Verordnung über die Errichtung einer gemeinsamen Marktorganisation für einen bestimmten Sektor erlassen worden ist, verpflichtet, sich aller Maßnahmen zu enthalten, die von dieser Verordnung abweichen oder ihr zuwiderlaufen. Mit einer gemeinsamen Marktorganisation sind auch Vorschriften unvereinbar, die deren ordnungsgemäßes Funktionieren behindern, auch wenn die gemeinsame Marktorganisation das betreffende Gebiet nicht abschließend geregelt hat $[\ldots]^{\text {*99 }}$.

In ähnlicher Weise führte der EuGH in seinem Urteil vom 14.10.2004 in der Rechtssache Spanien/Kommission aus: „Nach ständiger Rechtsprechung ist es Sache der [Union], sobald sie eine gemeinsame Marktorganisation für einen bestimmten Sektor errichtet hat, Lösungen für die im Rahmen der gemeinsamen Agrarpolitik auftretenden Probleme zu finden. Deshalb sind die Mitgliedstaaten verpflichtet, sich in diesem Bereich aller einseitigen Maßnahmen zu enthalten, selbst wenn diese geeignet sind, die gemeinsame Politik der Gemeinschaft zu unterstützen $[\ldots]^{\text {‘10 }}$.

\footnotetext{
7 EuGH, Urt. v. 23.1.1975, Rs. 51/74 (van der Hulst), Rn. 25-29.

${ }^{8}$ EuGH, Urt. v. 13.3.1984, Rs. 16/83 (Prantl), Rn. 13.

9 EuGH, Urt. v. 25.3.2004, Rs. C-118/02 (Industrias de Deshidratación), Rn. 19 f.

${ }^{10}$ EuGH, Urt. v. 14.10.2004, Rs. C-173/02 (Spanien/Kommission), Rn. 19.
} 
Speziell zur Frage einer nationalen Preisregelung meinte der EuGH in der mit Urteil vom 9.9.2003 entschiedenen Rechtssache Milk Marque, dass „die Mitgliedstaaten im Bereich einer gemeinsamen Marktorganisation - und erst recht dann, wenn diese auf einer gemeinsamen Preisregelung aufbaut - verpflichtet“ seien, „sich aller Maßnahmen zu enthalten, die von dieser Marktorganisation abweichen oder ihr zuwiderlaufen [...]. Insbesondere sind die Mitgliedstaaten nicht mehr befugt, durch einseitig erlassene innerstaatliche Rechtsvorschriften in den Mechanismus der Bildung derjenigen Preise einzugreifen, die in der gemeinsamen Marktorganisation auf der gleichen Produktions- oder Handelsstufe geregelt sind $[\ldots]^{\text {"11 }}$. Die ausführliche primärrechtliche Regelung zu den ausschließlichen und geteilten Kompetenzen der EU gab es zum Zeitpunkt dieser fünf Urteile noch nicht. Sie wurde erst durch den Vertrag von Lissabon mit Wirkung ab Dezember 2009 eingeführt.

Zuvor hatte der EuGH selbst die Rechtsfigur der ausschließlichen $\mathrm{Zu}-$ ständigkeit entwickelt und beispielsweise 1992 wie folgt beschrieben: „Ob die Zuständigkeit der [Union] ausschließlich ist, bestimmt sich nicht nur nach dem [EU-Primärrecht], sondern auch danach, in welchem Umfang die [Unionsorgane] zur Durchführung des [EU-Primärrechts] Maßnahmen getroffen haben; auf Grund solcher Maßnahmen können die Mitgliedstaaten Zuständigkeiten verlieren, die sie zuvor übergangsweise ausüben konnten“12. Diese Formulierung passt gut zur Ablösung der nationalen Agrarmarktordnungen durch die gemeinsame Agrarmarktorganisation, da die Mitgliedstaaten bis zum Abschluss der schrittweisen Errichtung der gemeinsamen Agrarmarktorganisation und damit übergangsweise für das Agrarmarktrecht noch zuständig gewesen sind.

\section{Die zwei Grundkonstellationen bei der Beurteilung nationaler Preisregelungen}

Werden die Fälle betrachtet, die hinter diesen beständig vom EuGH herangezogenen Grundsätzen stehen, so ergibt sich ein differenziertes Bild. Es lassen sich zwei Grundkonstellationen unterscheiden. In der ersten Grundkonstellation ist die GMO unmittelbar betroffen, indem es in der nationalen Regelung gleichzeitig um ein in der GMO geregeltes Agrarerzeugnis und eine von der GMO verfolgte Zielsetzung geht. Handelt es sich hingegen entweder um kein von der GMO erfasstes Erzeugnis oder um keine von der GMO verfolgte Zielsetzung, liegt die zweite Grundkonstellation vor.

${ }^{11}$ EuGH, Urt. v. 9.9.2003, Rs. C-137/00 (Milk Marque), Rn. 63.

12 EuGH, Gutachten 2/91 v. 19.3.1993 (IAO), Rn. 9. 
Die erste Grundkonstellation führt dazu, dass nur eine explizite oder implizite Befugnis zum Erlass nationalen Rechts, die in der GMO, in dem auf die GMO gestützten EU-Sekundärrecht oder in sonstigen Rechtsakten zum EU-Agrarmarkt enthalten ist, die Sperrwirkung der EU-Agrarmarktorganisation zu durchbrechen vermag. In solchen Konstellationen ist mithin zu ermitteln, ob das EU-Agrarmarktrecht die betreffende Frage abschließend regeln möchte oder mitgliedstaatliche Spielräume belässt. Liegt eine abschließende Regelung vor, ist nach herrschender Ansicht auf Grund des Art. 2 Abs. 2 Satz 2 AEUV der Erlass nationalen Rechts nicht mehr möglich. Nach der alternativen Argumentation zur ausschließlichen Zuständigkeit der EU für das Agrarmarktrecht sperrt hingegen bereits Art. 2 Abs. 1 Halbsatz 2 AEUV mitgliedstaatliches Recht.

Ist demgegenüber durch die nationale Regelung ein Erzeugnis betroffen, auf das die EU-Agrarmarktorganisation keine Anwendung findet, oder wird eine von der EU-Agrarmarktorganisation nicht umfasste Zielsetzung verfolgt, kehrt sich die Systematik um. Prinzipiell sperrt in dieser zweiten Grundkonstellation die EU-Agrarmarktordnung nationales Recht nicht. Allerdings darf das nationale Recht die Wirksamkeit des EU-Rechts nicht beeinträchtigen. Mithin ist zu prüfen, ob die Funktionsfähigkeit der EUAgrarmarktorganisation durch die nationale Regelung leidet. Zudem ist zu beachten, dass auch andere durch das EU-Recht gesetzte Schranken wie etwa der Grundsatz des freien Warenverkehrs oder grundrechtlicher Schutz eingreifen können.

\subsection{Die Rechtssache Industrias de Deshidratación: nicht erfasstes Erzeugnis}

Wird unter diesem Blickwinkel beispielhaft die erwähnte Rechtssache Industrias de Deshidratación betrachtet, so ging es um eine nationale Beihilfe für pflanzliches Frischfutter, bei dem unter anderem der Feuchtigkeitsgehalt höher sein durfte als bei pflanzlichem Trockenfutter. Dies stufte ein Produzent von pflanzlichem Trockenfutter als diskriminierend ein. Der EuGH befand, dass die nationale Regelung zu pflanzlichem Frischfutter nicht gegen die Gemeinsame Marktordnung für Trockenfutter verstieß, da dort pflanzliches Frischfutter nicht geregelt war und keine Anhaltspunkte dafür vorlagen, dass das Funktionieren der EU-Regelung beeinträchtigt wurde ${ }^{13}$. Folglich lag die zweite Grundkonstellation vor, die der EuGH gemäß der dargestellten Systematik prüfte.

\footnotetext{
13 EuGH (Fn. 9), Rn. 19 ff.
} 
Während die Frage, ob ein Erzeugnis von der EU-Agrarmarktorganisation - im Weiteren wird teils vereinfachend von der GMO gesprochen, obwohl neben ihr noch weitere Regelungen existieren - erfasst wird, regelmäßig eindeutig beantwortet werden kann, treten bei der Feststellung, ob die Zielsetzung, die von der nationalen Regelung verfolgt wird, mit einer von der GMO verfolgten Zielsetzung identisch ist, des Öfteren Schwierigkeiten auf. Zum einen lassen sich Zielsetzungen nicht immer eindeutig voneinander abgrenzen. Zum anderen ist nicht stets klar, ob die GMO eine bestimmte Zielsetzung verfolgt. In dieser Hinsicht seien im Folgenden vier EuGH-Fälle vorgestellt, in denen nach Ansicht des Verfassers eine mit der GMO verfolgte Zielsetzung vorlag und mithin das Prüfungsschema der ersten Grundkonstellation anzuwenden war.

\subsection{Die Rechtssache Lefèvre: Verbraucherinteressen}

Das chronologisch erste Beispiel ist das Urteil des EuGH vom 2.7.1987 in der Rechtssache Lefèvre. Dabei ging es um die nationale Festsetzung einer Handelsspanne, die bei dem Verkauf von Rindfleischerzeugnissen an den Verbraucher einzuhalten war. Offenbar sollten dadurch überhöhte Verbraucherpreise vermieden werden. Der EuGH sah eine solche Festsetzung als grundsätzlich möglich an, allerdings nur, soweit sie ,die Ziele und das Funktionieren der gemeinsamen Marktorganisation nicht" gefährdet. Im konkreten Fall stufte er diese Grenze als verletzt an, da die Handelsspanne anhand von pauschalen Parametern ermittelt wurde und daher nicht „die Beschaffungs- und Einfuhrkosten, die dem Einzelhändler tatsächlich entstanden sind“", berücksichtigte. Denn auf diese Weise würde für den Endverkäufer die Möglichkeit eingeschränkt, „sich beim Erzeuger oder Händler seiner Wahl einzudecken“, was „das Funktionieren der gemeinsamen Marktorganisation und insbesondere den [Handel innerhalb der EU]" beeinträchtige ${ }^{14}$. Die Prüfung des EuGH klingt so, als liege die zweite Grundkonstellation vor, obwohl das EU-Agrarmarktrecht nach Art. 39 Abs. 1 Buchstabe e AEUV die „Belieferung der Verbraucher $\mathrm{zu}$ angemessenen Preisen" mit verfolgen muss und folglich ein Vorliegen der ersten Grundkonstellation anzunehmen ist. Allerdings sind sowohl das Urteil als auch die zugehörigen Schlussanträge zu kurz gehalten, um die genaue Argumentation des EuGH aus der vorliegend betrachteten Perspektive rechtsdogmatisch nachvollziehen zu können.

${ }^{14}$ EuGH, Urt. v. 2.7.1987, Rs. 188/86 (Lefèvre), Rn. 11 ff. 


\subsection{Die Rechtssache Hammarsten: Gesundheitsschutz}

Viel klarer lässt sich demgegenüber dem Urteil des EuGH vom 16.1.2003 in der Rechtssache Hammarsten ein Vorliegen der ersten Grundkonstellation entnehmen. So sah die Gemeinsame Marktorganisation für Flachs und Hanf eine Beihilferegelung für Hanf vor. Dabei waren Vorkehrungen getroffen, um zu vermeiden, dass der Hanf zum Zwecke der Herstellung von Drogen verwendet wurde. Einem Mitgliedstaat ging dieser Schutz nicht weit genug. Er verbot daher den Anbau des entsprechenden Hanfes, so dass zugleich die Beihilferegelung nicht mehr in Anspruch genommen werden konnte. Der EuGH entschied hierzu in einem sehr kurzen Urteil, dass das nationale „Betäubungsmittelrecht kein Ziel des Allgemeininteresses“ verfolge, ,das nicht von der gemeinsamen Marktorganisation auf dem Hanfsektor abgedeckt wäre". Infolgedessen sei der Eingriff in die Gemeinsame Marktorganisation für Flachs und Hanf rechtswidrig ${ }^{15}$.

Hieran zeigt sich zugleich, dass eine Zielsetzung wie der Gesundheitsschutz, der nicht in Art. 39 AEUV genannt wird, trotzdem Bestandteil der EU-Agrarmarktorganisation sein kann. Denn der EU-Gesetzgeber ist verpflichtet, bei der Ausgestaltung der EU-Agrarmarktorganisation nicht nur die im Landwirtschaftstitel des AEUV niedergelegten Zielsetzungen, sondern auch andere Zielsetzungen der EU wie etwa den Gesundheits- und Tierschutz zu beachten. Dies kann sich dann wie im Falle des Hanfes in Agrarmarktregelungen zu einzelnen Erzeugnissen niederschlagen. Im Falle solcher punktuell aus anderweitigen Kapiteln des AEUV inkorporierten Zielsetzungen liegt ebenfalls die erste Grundkonstellation vor, wie die Rechtssache Hammarsten eindrücklich belegt.

\subsection{Die Rechtssache Milk Marque: Kartellrecht}

Eine wiederum aus dem Landwirtschaftstitel des AEUV selbst stammende Zielsetzung lag dem schon erwähnten Urteil des EuGH in der Rechtssache Milk Marque von 2003 zugrunde. Dabei ging es um den Eingriff einer nationalen Kartellbehörde in den Milchmarkt. Der Bereich des Kartellrechts bildete von Beginn an einen integralen Bestandteil der EU-Agrarmarktorganisation, so dass zu klären war, in welchem Umfang die EU-Agrarmarktorganisation einen solchen Eingriff gestattete. Mithin prüfte der EuGH aus der Perspektive der EU-Milchmarktordnung: „Da jedoch [...] die Aufrecht-

15 EuGH, Urt. v. 16.1.12003, Rs. C-462/01 (Hammarsten), Rn. 31 ff. 
erhaltung eines wirksamen Wettbewerbs zu den Zielen der gemeinsamen Marktorganisation für Milch und Milcherzeugnisse gehört, ist zum einem festzustellen, dass nicht jede Maßnahme, die die Behörden eines Mitgliedstaats nach dem nationalen Wettbewerbsrecht erlassen, ihrem Wesen nach als Abweichung von der gemeinsamen Marktorganisation oder als Beeinträchtigung ihrer Funktionsweise bewertet werden kann. Zum anderen können nationale Maßnahmen zur Beseitigung einer Wettbewerbsverzerrung, die aus dem Missbrauch der Machtstellung einer Agrarkooperative auf dem nationalen Markt entstanden ist, nicht von vornherein als Maßnahmen eingestuft werden, die [...] in den Mechanismus der Bildung derjenigen Preise eingreifen, die durch die gemeinsame Marktorganisation auf [Unionsebene] geregelt werden“. Hinzu komme, dass - so der EuGH weiter - bei der Annahme, im Anwendungsbereich des Landwirtschaftskapitels das AEUV seien sämtliche Handlungen nationaler Wettbewerbsbehörden untersagt, eine „Wettbewerbsverzerrung ohne [unionsweite] Bedeutung durch überhaupt keine Maßnahme ausgeräumt werden" könne ${ }^{16}$.

EU-primärrechtlich steht hinter diesem Urteil, dass Art. 42 Unterabs. 1 AEUV die Anwendung des allgemeinen EU-Kartellrechts im Bereich der Landwirtschaft von einer Entscheidung des EU-Gesetzgebers abhängig macht. Im Rahmen der EU-Agrarmarktorganisation hat der EU-Gesetzgeber eine solche Anwendungsentscheidung getroffen, die lediglich unter dem Vorbehalt spezieller Regelungen im EU-Agrarrecht steht. Soweit das EUAgrarmarktrecht den Wettbewerb nicht beschränkt, sollen die Marktteilnehmer folglich untereinander in Wettbewerb stehen. Als Folge kann die Europäische Kommission als EU-Kartellbehörde grundsätzlich auch auf dem Feld des Agrarmarktes tätig werden, um gegen grenzüberschreitende Wettbewerbsbeschränkungen vorzugehen. Daher ist es nur konsequent, dass bei rein nationalen Sachverhalten die nationalen Kartellbehörden ebenso handeln dürfen. Denn den verbliebenen, von der EU-Agrarmarktorganisation gewollten Wettbewerb im Agrarbereich zu schützen, ist mit Aufgabe der Kartellbehörden.

Die Kartellbehörden müssen dabei zum einen die im EU-Agrarmarktrecht enthaltenen Kartellbefreiungen berücksichtigen und dürfen zum anderen die Instrumente der EU-Agrarmarktorganisation nicht beeinträchtigen. Genau dies hat der EuGH im konkreten Fall geprüft und ist zu dem Ergebnis gekommen, dass die streitige Anordnung der nationalen Kartellbehörde nicht das Funktionieren der EU-Milchmarktordnung beeinträchtigt hat. Sie greife nicht in die Preisbildung ein, sondern gewährleiste genau im Gegenteil ,das ordnungsgemäße Funktionieren der Preismechanismen [...], um ein Preisni-

16 EuGH (Fn. 11), Rn. 64 f. 
veau zu erreichen, dass“ - hier nimmt der EuGH indirekt auf die Ziele des Art. 39 AEUV Bezug - ,sowohl den Interessen der Erzeuger als auch denen der Verbraucher dient" ${ }^{\text {"17 }}$. Folglich stimmte die Entscheidung des nationalen Gesetzgebers, ein Eingreifen der nationalen Kartellbehörde zu ermöglichen, mit den Zielsetzungen der EU-Agrarmarktorganisation überein.

\subsection{Die Rechtssache Kuipers: Qualitätsnormen}

Als letztes Beispiel für die erste Grundkonstellation sei das Urteil des EuGH vom 26.5.2005 in der Rechtssache Kuipers angeführt. In ihm ging es wie in der Rechtssache Lefèvre um eine nationale Preisregelung. Ein nationales Gesetz hatte den nationalen Milchbranchenverband ermächtigt, ein Qualitätssystem zu errichten, wonach bei Unterschreitung einer bestimmten Rohmilchqualität ein Abschlag vom Kaufpreis für die Rohmilch vorgenommen werden musste. Die so einbehaltenen Beträge waren im Gegenzug für einen Zuschlag zu verwenden, den Rohmilch erhielt, die die festgelegte Rohmilchqualität übertraf. Die Höhe der Abschläge und Zuschläge konnten dabei von den Abnehmern nicht selbst individuell festgelegt werden. Vielmehr erfolgte die Festlegung der Höhe des Abschlages einheitlich für das gesamte Hoheitsgebiet.

So wurde aus den jeweils für zwölf Monate einbehaltenen Abschlägen ein Gesamtbetrag errechnet, der auf die in diesem Zeitraum mit Zuschlägen zu versehene Rohmilch aufzuteilen war. Dadurch ergab sich eine ebenfalls einheitliche Höhe des Zuschlages. Behielt ein Abnehmer mehr Abschläge ein, als er Zuschläge zahlte, musste er den Differenzbetrag an eine zentrale Stelle überweisen, von der aus die überschüssigen Beträge an Abnehmer gingen, die mehr Zuschläge zu zahlen hatten, als von ihnen Abschläge einbehalten worden waren. Der Milchbranchenverband errichtete ein derartiges Zwangssystem. Folglich entstand ein zentral gesteuerter, nationalweit gültiger Qualitätsausgleich, der einen Eingriff in die freie Gestaltung der Rohmilchpreise darstellte.

Ein Milcherzeuger wehrte sich gegen einen Abschlag, woraufhin das nationale Gericht dem EuGH unter anderem die Frage vorlegte, ob die Preisregelung mit der EU-Milchmarktordnung vereinbar war. Der EuGH entschied, dass die Mitgliedstaaten zwar Qualitätskriterien für Rohmilch regeln können, die Verbindung mit einem derartigen Preissystem jedoch als „Mechanismus der Bildung der Preise“ unzulässig sei: „Diese Regelung verpflichtet nämlich

17 EuGH (Fn. 11), Rn. 86. 
sämtliche Molkereibetriebe, in einem Fall einen Abschlag vom normalerweise an die Milchviehhalter zu zahlenden Preis einzubehalten [...], im anderen Fall [...] den Haltern [...] einen Zuschlag zu zahlen, der zu dem dem jeweiligen Halter normalerweise zu zahlenden Preis hinzugerechnet wird und über die Gesamtheit der von den niederländischen Molkereibetrieben auf Grund der Lieferung von qualitativ minderwertiger Milch einbehaltenen Abschläge finanziert wird. Die genannte Regelung legt also im Voraus bestimme Faktoren fest, die bei der endgültigen Festsetzung des Preises eine Rolle spielen, den die Molkereibetriebe den Milchviehhaltern, die ihnen Milch liefern, zu zahlen haben." Außerdem müssten die Abnehmer, die mehr Rohmilch von geringer als von höherer Qualität geliefert erhielten, die Abschläge abführen. Sie blieben so auf den Kosten für die geringere Qualität sitzen, ohne für die geringere Qualität verantwortlich zu sein ${ }^{18}$.

Eine möglichst hohe Qualität der Agrarerzeugnisse zu erreichen, ist eine Zielsetzung der EU-Agrarmarktorganisation, wie das Vermarktungsnormenkapitel der GMO zeigt. Schon früh entstanden in den meisten Erzeugnisbereichen Normen, die eine einheitlich hohe Qualität der Erzeugnisse sicherstellen sollten. Insbesondere im Milchbereich haben solche Bestimmungen eine jahrzehntelange Tradition. Dadurch sollen die Absatzmöglichkeiten verbessert, der Binnenhandel erleichtert und eine Standardqualität für Interventionsware gewährleistet werden. Wie der EuGH hervorhob, war ursprünglich auch eine qualitätsbezogene Bezahlung von Rohmilch vorgesehen, wurde jedoch nie implementiert. Daraus wird allgemein geschlossen, dass die Mitgliedstaaten befugt sind, Regelungen zur Sicherstellung der Qualität von Rohmilch ${ }^{19} \mathrm{zu}$ erlassen, die den Preisaspekt mit einschließen, soweit sie dadurch das Funktionieren der EU-Milchmarktordnung nicht beeinträchtigen.

Der EuGH befasste sich nicht näher mit der Frage, ob die Zielsetzung der nationalen Regelung grundsätzlich unter die EU-Agrarmarktorganisation fiel, sondern hielt lediglich fest, dass die Mitgliedstaaten im Bereich der Qualität von Rohmilch regelungsbefugt sind und dabei auch den Aspekt der Bezahlung normieren dürfen. Diesen Regelungsfreiraum grenzte er anschließend durch die EU-Milchmarktordnung ein. Nach Ansicht des Verfassers lag auch in diesem Fall vor dem Hintergrund der umfangreichen Vermarktungsnormen der EU-Agrarmarktorganisation ein Fall der ersten Grundkonstellation vor. Hierzu sei noch Erwägungsgrund 65 der GMO zitiert, der besagt: „Die

${ }^{18}$ EuGH, Urt. v. 26.5.2005, Rs. C-283/03 (Kuipers), Rn. 41 ff. Der Verfasser war an dem Verfahren auf Seiten der Europäischen Kommission beteiligt.

${ }_{19}$ Zur Klarstellung sei bemerkt, dass es dabei nicht um die lebensmittelrechtlichen Mindestanforderungen an Rohmilch geht. Diese sind gesondert im EU-Lebensmittelhygienerecht einheitlich für alle Mitgliedstaaten festgelegt. 
Anwendung von Normen für die Vermarktung landwirtschaftlicher Erzeugnisse kann zu einer Verbesserung der wirtschaftlichen Bedingungen für die Erzeugung und Vermarktung sowie der Qualität dieser Erzeugnisse beitragen. Die Anwendung solcher Normen liegt daher im Interesse der Erzeuger, der Händler und der Verbraucher".

Generalanwältin Kokott war in ihren Schlussanträgen vom 16.12.2004 zur Zulässigkeit der nationalen Regelung gekommen, da die EU-Milchmarktordnung ,keine eigenen Bestimmungen zur qualitätsorientierten Bezahlung von Rohmilch“" enthalte und die EU ebenfalls eine hohe Qualität von Rohmilch anstrebe. Hierbei nahm sie insbesondere auf die Zielsetzungen des Art. 39 Abs. 1 Buchstabe c und e AEUV Bezug und ordnete somit das von der nationalen Regelung verfolgte Ziel - neben dem ebenfalls von ihr genannten Gesundheitsschutz - der EU-Agrarmarktorganisation $\mathrm{zu}^{20}$. Ihre Argumentation übersah jedoch anschließend, dass zur Erreichung einer qualitätsorientierten Bezahlung ein derart tiefer Eingriff in die freie Preisbildung nicht erforderlich ist. So legt beispielsweise die deutsche Qualitätsregelung über die Bezahlung der Rohmilch nur fest, dass der Abnehmer im Falle von Rohmilch geringerer Qualität einen Mindestabschlag vornehmen muss. $\mathrm{Ob}$ er den Abschlag höher ansetzt und wie er mit den einbehaltenen Beträgen umgeht, ist ihm überlassen ${ }^{21}$. Dadurch verbleibt erstens der finanzielle Ausgleich für die Lieferung minderwertigerer Rohmilch bei dem betreffenden Abnehmer. Zweitens kann er selbst entscheiden, ob er ihn für Zuschläge bei höherwertiger Rohmilch verwendet oder beispielsweise in ein Beratungssystem investiert, um seine Lieferanten dabei zu unterstützen, die Ursachen für die minderwertige Rohmilch zu ermitteln und abzustellen.

\subsection{Die Rechtssache Scotch Whisky Association: Gesundheitsschutz}

Mithin verstieß die nationale Regelung in den Rechtssachen Hammarsten, Kuipers und Lefèvre gegen EU-Agrarmarktrecht und vermochte daher keinen Bestand zu haben, während in der Rechtssache Milk Marque kein derartiger Verstoß vorlag. In allen vier Fällen konnte festgestellt werden, dass die jeweilige Zielsetzung der nationalen Regelung - Gesundheitsschutz, Qualität, Verbraucherpreisniveau und Wettbewerb - auch von der EU-Agrarmarktorganisation verfolgt wurde, so dass geprüft werden musste, ob die EU-Agrarmarktorganisation einen Regelungsfreiraum beließ und bei

20 J. Kokott, Schlussanträge v. 16.12.2004, Rs. C-283/03 (Kuipers), Rn. 34 ff.

${ }^{21} \S \S 30 \mathrm{ff}$. der Verordnung zur Förderung der Güte von Rohmilch (Rohmilchgüteverordnung - RohmilchGütV) v. 11.1.2021, Bundesgesetzblatt 2021, Teil I, S. 47. 
Bejahung dieser Frage, ob die nationale Ausgestaltung dieses Freiraumes mit Regelungen der EU-Agrarmarktorganisation kollidierte. In der Rechtssache Hammarsten wurde schon der Regelungsfreiraum verneint, während in den anderen drei Rechtssachen die Entscheidung über die Rechtmäßigkeit der nationalen Regelung erst im Rahmen der Kollisionsprüfung fiel.

Angesichts der weitgesteckten Zielsetzungen der EU-Agrarmarktorganisation, die teilweise über die in Art. 39 AEUV genannten Zielsetzungen hinausgehen, sind Fälle, in denen eine ausschließlich anderweitige Zielsetzung durch die nationale Regelung verfolgt wird, eher selten. Einen solchen Fall entschied der EuGH mit Urteil vom 23.12.2015 in der Rechtssache Scotch Whisky Association. Zum Schutz vor Alkoholmissbrauch und damit der Gesundheit waren Mindestpreise für den Verkauf von Alkohol an Verbraucher festgesetzt worden. Insofern lag eindeutig eine Preisregelung vor, deren Vereinbarung mit der EU-Weinmarktordnung zu klären war.

Der EuGH stellte fest, dass die in der GMO geregelte EU-Weinmarktordnung keinen Preismechanismus enthält und daher, ,die freie Bestimmung der Verkaufspreise auf der Grundlage des freien Wettbewerbs" regele. Allerdings würde die GMO mit Wein verbundene Gesundheitsgefahren nicht thematisieren. Demnach könnten die Mitgliedstaaten diesen Aspekt normieren und dafür auch auf eine Preisregelung zurückgreifen, falls dadurch der freie Wettbewerb auf dem Weinmarkt nicht unverhältnismäßig eingeschränkt werde. Wörtlich äußerte der EuGH: „Insoweit ist festzustellen, dass die Schaffung einer GMO es den Mitgliedstaaten tatsächlich nicht verwehrt, nationale Regelungen anzuwenden, die ein im Allgemeininteresse liegendes anderes Ziel als die von der betreffenden GMO erfassten Ziele verfolgt, selbst wenn diese Regelung einen Einfluss auf das Funktionieren des Gemeinsamen Marktes im betreffenden Wirtschaftsbereich hat (vgl. Urteil Hammarsten ...). Demnach darf sich ein Staat auf das Ziel berufen, die Gesundheit und das Leben von Menschen zu schützen, um eine Maßnahme [...] zu rechtfertigen, die das System der freien Preisbildung unter wirksamen Wettbewerbsbedingungen beeinträchtigt, auf das sich die Verordnung (EU) Nr. 1308/2013 gründet". Der Verweis auf die Rechtssache Hammarsten war allerdings insofern missverständlich, als dort gerade kein ,anderes Ziel“ verfolgt wurde.

Im Weiteren führte der EuGH eine klassische Verhältnismäßigkeitsprüfung durch und äußerte Zweifel, ob steuerliche Maßnahmen nicht denselben Effekt hätten, jedoch weniger eingreifend seien. Letztlich überließ es der EuGH dem vorlegenden nationalen Gericht, dies zu bewerten ${ }^{22}$. Generalanwalt Bot war in seinen Schlussanträgen vom 3.9.2015 unter anderem noch

${ }^{22}$ EuGH, Urt. v. 23.12.2015, Rs. C-313/14 (Scotch Whisky Association), Rn. 26 ff. 
näher auf den Aspekt eingegangen, ob die GMO auch die Zielsetzung des Gesundheitsschutzes verfolge. Zwar beziehe die GMO den „Schutz der Gesundheit von Mensch oder Tier“ in „,mehreren Bestimmungen“ ein, ohne dass es jedoch dadurch „Aufgabe der Verordnung“ sei, „auf Unionsebene das Ziel des Gesundheitsschutzes im Allgemeinen und dasjenige der Bekämpfung des gefährlichen oder übermäßigen Konsums alkoholischer Getränke im Besonderen zu gewährleisten. Obwohl der Schutz der Gesundheit ein tatsächliches Ziel der [Gemeinsamen Agrarpolitik] ist, ist er daher trotzdem von untergeordneter Bedeutung, so dass dieses Ziel von den Mitgliedstaaten zur Rechtfertigung einer nationalen Regelung, die Auswirkungen auf das Funktionieren der GMO in dem betroffenen Bereich hat, geltend gemacht werden kann“. Zudem schlösse Art. 168 Abs. 5 AEUV ,jegliche Harmonisierung der Rechts- und Verwaltungsvorschriften der Mitgliedstaaten zum Schutz und zur Verbesserung der menschlichen Gesundheit" aus. Auf andere Bestimmungen des EU-Primärrechts für eine Harmonisierung zurückzugreifen, würde eine unzulässige Umgehung des Art. 168 Abs. 5 AEUV bedeuten ${ }^{23}$.

Diese Überlegungen erscheinen zu pauschal. Der EuGH hat sie denn auch nicht übernommen. Denn die Pflicht der EU, die Gesundheit von Menschen zu schützen, fließt vor allem aus dem grundrechtlichen Schutz der Gesundheit, wie er unter anderem in Art. 3 Abs. 2 der EU-Grundrechtecharta zu enthalten ist. Mithin muss die EU bei der Ausgestaltung ihrer Agrarmarktorganisation gesundheitsbezogene Aspekte berücksichtigen, falls eine Maßnahme im Rahmen der EU-Agrarmarktorganisation zu einer Gesundheitsgefährdung führen kann. Folglich ist die Ansicht von Generalanwalt Bot dahingehend einzuschränken, dass das Landwirtschaftskapitel des AEUV nicht dazu dienen kann, genuine Rechtsharmonisierung im Bereich der menschlichen Gesundheit zu betreiben. Ist die gesundheitsschützende Regelung hingegen - so wie in der Rechtssache Hammarsten - in ein Instrument der EU-Agrarmarktorganisation eingebettet, dürfen die Mitgliedstaaten keine davon abweichende gesundheitsschützende Regelung treffen, falls durch diese abweichende Regelung das Funktionieren des EU-Agrarmarktinstruments beeinträchtigt wird.

\section{Die Rechtssache Lietuvos Respublikos Seimo: Bekämpfung unfairer Handelspraktiken}

2019 erging das Urteil des EuGH in der Rechtssache Lietuvos Respublikos Seimo, das zu der Frage zurückführt, wie die jeweils verfolgten Zielsetzungen $\mathrm{zu}$ bestimmen und voneinander abzugrenzen sind. In Li-

${ }^{23}$ Y. Bot, Schlussanträge v. 3.9.2015, Rs. C-313/14 (Scotch Whisky Association), Rn. 41 ff. 
tauen herrschte damals gemäß der Darstellung der litauischen Regierung ein starkes Machtungleichgewicht auf dem Rohmilchmarkt. Sechs Verarbeitungsunternehmen nahmen fast die gesamte litauische Rohmilch ab, die von 20.000 teils sehr kleinen Milcherzeugungsbetrieben stammte. Die Milcherzeugungsbetriebe waren untereinander kaum organisiert. Der Preis für die Rohmilch wurde von den Verarbeitungsunternehmen auf Grund ihrer Marktmacht einseitig ohne Vertragsverhandlungen festgesetzt. Litauen regelte daraufhin, dass - vereinfacht gesprochen - jedem Milcherzeuger derselbe Mindestpreis gezahlt werden musste. Die Höhe dieses Mindestpreises richtet sich nach dem höchsten Preis, der einem Milcherzeuger gezahlt wurde. Zahlte mithin ein Abnehmer einen höheren Preis als die anderen Abnehmer, mussten sich alle Abnehmer nach diesem höheren Preis richten. Ausgenommen wurden nur Milcherzeuger, die in einer staatlich anerkannten Milcherzeugerorganisation zusammengeschlossen waren, wobei auch diesen Milcherzeugern mindestens der für die nicht organisierten Milcherzeuger festgelegte Preis gezahlt werden musste. Im Endeffekt legte Litauen dadurch einen Mindestpreis für Rohmilch fest, der für alle Milcherzeuger und Abnehmer in Litauen galt ${ }^{24}$.

\subsection{Das Urteil des EuGH vom 13.11.2019}

Der Hauptdiskussionspunkt in der Rechtssache war, ob die Zielsetzung der nationalen Preisregelung mit einer Zielsetzung der GMO übereinstimmte und damit im Lichte der vorliegend entwickelten Systematik, ob die erste oder die zweite Grundkonstellation gegeben war. Litauen begründete die Regelung mit der Bekämpfung unfairer Handelspraktiken. Der EuGH stellte zunächst dar, dass die GMO an mehreren Stellen unfaire Handelspraktiken thematisierte, meinte jedoch, dass das ,Ziel, unfaire Praktiken zu bekämpfen“, von der GMO nicht abgedeckt sei, ,zumal diese Praktiken in der Verordnung weder als Ganzes erfasst noch von ihr geregelt, ja nicht einmal konkret benannt werden" ${ }^{25}$. Dass unfaire Handelspraktiken überhaupt nicht von der GMO geregelt werden, stimmt indes nicht. So führte der EuGH zuvor selbst aus, dass die in Art. 148 GMO für den Milchbereich enthaltene und für die Mitgliedstaaten optionale Vertragsregulierung dazu beitragen soll, „'bestimmte unfaire Handelspraktiken' zu unterlassen" ${ }^{\text {"26. }}$.

${ }^{24}$ EuGH, Urt. v. 13.11.2019, Rs. C-2/18 (Lietuvos Respublikos Seimo), Rn. 38. Der Verfasser war an dem Verfahren auf Seiten der deutschen Bundesregierung beteiligt.

${ }^{25}$ EuGH (Fn. 24), Rn. 49.

${ }^{26} \mathrm{EuGH}$ (Fn. 24), Rn. 48. 
In der Tat möchte die EU mit Art. 148 GMO und dem parallelen Art. 168 GMO erreichen, dass jeder Erzeuger über einen schriftlichen Vertrag mit seinem Abnehmer verfügt und dieser Vertrag konkrete Mindeststandards erfüllt. Auf diese Weise sollen die Erzeuger vor einseitigen Handlungen der Abnehmer geschützt werden und Rechtssicherheit erhalten. Beide Artikel verwenden zwar nicht den Terminus ,unfaire Handelspraktiken“, sind jedoch inhaltlich und ausweislich der zugehörigen Erwägungsgründe unter anderem auf die Vermeidung solcher Praktiken ausgerichtet. Da es vorrangig auf den materiellen Gehalt einer Regelung und nicht auf die - angesichts der zahlreichen Sprachfassungen ohnehin nicht immer vollständig treffend - verwendete Terminologie ankommt, überzeugt den Verfasser die Argumentation des EuGH nicht.

Des Weiteren übergeht der EuGH den Zusammenhang zum Kartellrecht und damit verbunden zu dem in der GMO enthaltenen Recht der anerkannten Agrarorganisationen. Um die Marktmacht der Erzeugerseite zu stärken, stellt die GMO das Instrument der anerkannten Erzeugerorganisation zur Verfügung, mit deren Hilfe die Erzeugerseite weitreichend vom allgemeinen Kartellverbot befreit wird. Dass die Milcherzeuger in Litauen dieses Instrument, dessen Nutzung die EU zudem finanziell fördert, nicht in Anspruch genommen haben, kann nicht dazu führen, dass es aus der Gesamtbetrachtung auszublenden ist. Es sei an die Rechtssache Hammarsten erinnert, in der ein Mitgliedstaat die EU-Agrarmarktregelung für nicht tauglich erachtete und stattdessen eine eigene Regelung erließ, die der EU-Agrarmarktregelung widersprach. Könnte ein Mitgliedstaat die Anwendung einer EU-Agrarmarktregelung jederzeit durch eine eigene ersetzen, würde dies die EU-Agrarmarktorganisation in ihren Grundfesten erschüttern. Stuft ein Mitgliedstaat die EU-Agrarmarktorganisation in einem bestimmten Punkt als nicht zielführend oder ausreichend ein, muss er versuchen, eine Änderung der EU-Agrarmarktorganisation zu erreichen.

Anders gewendet hat die Liberalisierung des EU-Agrarmarktes dazu geführt, dass nun weitgehend das Wettbewerbsprinzip herrscht, wie auch der EuGH anführt. In Art. 148 Abs. 4 Unterabs. 1 GMO als einer explizit auf den Milchmarkt ausgerichteten Bestimmung heißt es sogar ausdrücklich: „Sämtliche Bestandteile von Verträgen über Rohmilchlieferungen [...] sind zwischen den beteiligten Parteien frei verhandelbar". Hierzu gehört gemäß Art. 148 Abs. 2 Buchstabe c GMO unter anderem der Preis. Um im Rahmen des Wettbewerbsprinzips für ausgewogene Bedingungen zu sorgen, hält die GMO verschiedene Instrumente bereit. Dazu zählen unter anderem die Vertragsregulierung und die Kartellfreistellung für anerkannte Erzeugerorganisationen. Daneben gilt über die Anwendungsanordnung des 
Art. 206 Unterabs. 1 GMO das in Art. 102 AEUV geregelte Verbot des Missbrauchs einer marktherrschenden Stellung.

Die GMO gestaltet folglich die Wettbewerbsregeln auf dem EU-Agrarmarkt aktiv aus, so dass nach Ansicht des Verfassers in der Rechtssache Lietuvos Respublikos Seimo nicht wie vom EuGH vertreten die zweite Grundkonstellation, sondern die erste Grundkonstellation vorlag. Folglich war Litauen der - als flächendeckender Mindestpreis äußerst gravierende Eingriff in die Preisgestaltung auf dem Milchmarkt grundsätzlich verwehrt. Dies führt dazu, nach einer expliziten oder impliziten Regelungsbefugnis für die Mitgliedstaaten in der GMO und damit einer Ausnahme von dem Verbot zu suchen. Eine solche Ausnahme ist jedoch nicht ersichtlich. Damit ist nicht gesagt, dass den Mitgliedstaaten generell nationale Regelungen zur Bekämpfung unfairer Handelspraktiken durch die GMO verboten sind. Sofern sie mit keinen Instrumenten oder Grundprinzipien der GMO - wie im konkreten Fall mit dem freien Wettbewerb um den Preis - kollidieren, dürften sie prinzipiell zulässig sein, um das Wettbewerbsprinzip abzusichern.

Allerdings setzt ihre Nutzung die Feststellung voraus, dass die in der GMO vorhandenen Instrumente nicht ausreichend sind, um das jeweils verfolgte Ziel zu erreichen. Auch diesbezüglich stößt die Regelung Litauens auf Schwierigkeiten. So hat Litauen nicht dargelegt, warum die Problematik nicht durch eine Anwendung des kartellrechtlichen Missbrauchstatbestands sowie der Vertragsregulierung mit ihrer Pflicht zur schriftlichen vertraglichen Einigung über den Preis und folglich mit Hilfe von aus der GMO fließender Instrumente gelöst werden konnte. Der EuGH bemerkte zwar zu dem letzteren Punkt, dass Art. 148 GMO nur den Vertragsabschluss, nicht jedoch spätere einseitige Änderungen des Vertrages betreffe ${ }^{27}$. Einseitige Änderungen eines Vertrages und insbesondere die einseitige Änderung des Kaufpreises dürften jedoch auch nach dem litauischen Zivilrecht gegen den Grundsatz des pacta sunt servanda verstoßen. Falls das Problem in einer vertragswidrigen Handlung liegt, wäre das viel geringer in die GMO eingreifende Instrument eine staatliche Überwachung der Vertragsdurchführung.

Genau dieses Instrument hat die EU deshalb auch im Rahmen der auf das Landwirtschaftskapitel des AEUV gestützten Richtlinie (EU) 2019/633 zur Bekämpfung unfairer Handelspraktiken in der Agrar- und Lebensmittelversorgungskette gewählt. So verpflichtet der dortige Art. 3 Abs. 1 Buchstabe c die Mitgliedstaaten, die einseitige Änderung eines vereinbarten Preises durch den Abnehmer zu verbieten. Nach Art. 4 ff. muss ein mitgliedstaatliches System der Überwachung der Einhaltung des Verbotes errichtet werden,

${ }^{27}$ EuGH (Fn. 24), Rn. 76. 
das zudem die Verhängung von Sanktionen ermöglicht. Der EuGH zog zwar die Richtlinie (EU) 2019/633 als Beleg dafür heran, dass die Bekämpfung unfairer Handelspraktiken in der GMO nicht abschließend geregelt is $\mathrm{t}^{28}$, nutzte den Inhalt der Richtlinie (EU) 2019/633 jedoch nicht zur Überprüfung der Verhältnismäßigkeit der litauischen Regelung. Die Richtlinie (EU) 2019/633 bietet mit dem vertraulichen behördlichen Verfahren auch eine Lösung für das vom EuGH angesprochene Retorsionsproblem ${ }^{29}$ an.

\subsection{Die Schlussanträge von Generalanwalt Bobek vom 7.3.2019}

In seinen zugehörigen Schlussanträgen meinte Generalanwalt Bobek, dass die „Weiterentwicklung der Regelung der Gemeinsamen Agrarpolitik“ die Frage der Zulässigkeit nationaler Maßnahmen ,in ganz neuen Licht erscheinen“ lassen würde: „Die schrittweisen Reformen der Gemeinsamen Agrarpolitik haben [...] zu einem mehr marktorientierten Ansatz geführt, der darauf abzielt, vor dem Hintergrund der Globalisierung des Handels die Wettbewerbsfähigkeit und Zukunftsfähigkeit des Sektors zu stärken. Diese Ansatzänderung hatte wiederum zur Folge, dass der Umfang des Vorrang gegenüber dem Spielraum für die einzelstaatliche Gesetzgebung 'rekalibriert' wurde. Inzwischen basiert die einheitliche gemeinsame Marktorganisation nicht mehr auf einem gemeinsamen Preissystem. Man kann also sagen, dass diese Elemente (in vollem Umfang) wieder der allgemeinen Regelung der geteilten Zuständigkeit unterliegen. Gemäß Art. 4 Abs. 2 Buchstabe d AEUV ist die Gemeinsame Agrarpolitik ein Bereich, der in die geteilte $\mathrm{Zu}$ ständigkeit der Europäischen Union und der Mitgliedstaaten fällt‘ ${ }^{\star 30}$. Diese Argumentation überzeugt den Verfasser nicht. Auch vor der Agrarmarktliberalisierung lag eine geteilte Zuständigkeit vor. Lediglich die Intensität der EU-Agrarmarktregelungen war höher. Nicht angesprochen wird zudem die Frage, ob im Bereich der EU-Agrarmarktorganisation eine ausschließliche Zuständigkeit gegeben ist, die die von Bobek selbst erwähnte totale Sperrwirkung für Preisregelungen im vorherigen nichtliberalisierten System erklären würde.

Der Verfasser ist der Auffassung, dass die Frage der Zulässigkeit nationaler Maßnahmen im Bereich der EU-Agrarmarktorganisation durch die Marktliberalisierung in keinem ,ganz neuen Licht“ erscheint. Vielmehr

${ }^{28} \mathrm{EuGH}$ (Fn. 24), Rn. 52 ff.

${ }^{29}$ EuGH (Fn. 24), Rn. 79.

${ }^{30}$ M. Bobek, Schlussanträge v. 7.3.2019, Rs. C-2/18 (Lietuvos Respublikos Seimo), Rn. 19 und 23 . 
kann wie vorliegend dargestellt die aus der Zeit vor der Marktliberalisierung stammende Systematik auch für die Zeit danach verwendet werden. Denn weder primärrechtlich noch sekundärrechtlich wollte die EU mit der Liberalisierung der EU-Agrarmarktordnung nationale Preisregelungen oder sonstige marktsteuernde Mechanismen wieder zulassen. Nationale Marktordnungen bleiben schon auf der primärrechtlichen Ebene weiterhin untersagt, sofern ihre Errichtung nicht ausdrücklich durch die EU-Agrarmarktorganisation gestattet wird. Diese Gestattung muss sich an den Zielen des Art. 39 AEUV orientieren.

Zugestimmt werden kann hingegen den Ausführungen von Bobek zu der Konstellation, dass die EU-Agrarmarktorganisation und die nationale Regelung dieselbe Zielsetzung verfolgen: „Der Unionsgesetzgeber hat sich nach Abwägung aller relevanten Faktoren für eine bestimmte normative Lösung entschieden, die einen Ausgleich der verschiedenen Interessen und zu berücksichtigenden Erwägungen erfordert. Verfolgt eine nationale Vorschrift dieselben Ziele durch eine andere normative Regelung, so liegt dem eine andere Bewertung desselben Gegenstands zugrunde“. Gewendet auf die konkrete Rechtssache bedeutet dies, dass die EU für die Frage der einseitigen Festlegung des Rohmilchpreises durch den Abnehmer in der GMO die Lösung vorsieht, dass jeder Milcherzeuger über einen schriftlichen Vertrag mit dem Abnehmer verfügen muss, der den Preis vor der Lieferung der Rohmilch festlegt, und die Mitgliedstaaten die Einhaltung dieser Regelung überwachen. Litauen hat sich für eine andere Lösung entschieden, die mit der Lösung der EU kollidiert. Denn die litauische Lösung greift in direkt in den Vertragspreis für Rohmilch ein, dessen freie Verhandelbarkeit nach der Lösung der EU jedoch gerade unangetastet bleiben soll.

Genau zu diesem Schluss kam Bobek denn auch, indem er die litauische Regelung als gegen Art. 148 GMO verstoßend und damit als unzulässig einstufte. Den Grundsatz, dass ein Mitgliedstaat eine in der EU-Agrarmarktorganisation vorgesehene Lösung für ein Problem nicht schlicht verwerfen und an deren Stelle eine eigene Lösung setzen darf, formulierte Bobek in folgender plakativer Weise, wobei er mit feiner Ironie auf ein Milcherzeugnis aus einem Drittstaat Bezug nahm: „Wenn allerdings die Marktorganisation für landwirtschaftliche Erzeugnisse nicht so löchrig werden soll wie ein Schweizer Käse, kann den Mitgliedstaaten [...] nicht gestatten werden, einseitig von den klaren Anforderungen des Unionsrechts abzuweichen [...] Wenn sich für die Mitgliedstaaten beim Vollzug Schwierigkeiten und Probleme ergeben, die ihres Erachtens behebungsbedürftig sind, so stehen ihnen die auf der Unionsebene vorgesehenen institutionellen Wege offen, um auf Gesetzesänderungen und die fortlaufende Anpassung an die sich verändern- 
den Gegebenheiten hinzuwirken“. Spiegelbildlich sei die EU verpflichtet, auf neue Entwicklungen zu reagieren. Falls sich ein von der EU geregeltes Instrument der EU-Agrarmarktorganisation als nicht mehr geeignet erweise, um das betreffende Problem zu bewältigen, fordere der Grundsatz der Verhältnismäßigkeit, dass es angepasst oder aufgehoben wird: „Andernfalls könnte [seine] Gültigkeit angegriffen werden. Ein solches Verfahren wäre jedoch ein eigener, abweichende Ausführungen und Beweise vor dem [EuGH] erfordernder Fall“331.

\section{Die Rechtssache Kommission/Ungarn: Verbot von Preisdifferenzierungen}

Das jüngste Urteil des EuGH in Bezug auf die vorliegend erörterte Thematik stammt vom 11.3.2021 in der Rechtssache Kommission/Ungarn. Ungarn ordnete - wiederum vereinfacht dargestellt - für ungarische Einzelhändler durch Gesetz an, dass in ihrer Zusammensetzung und in ihren organoleptischen Eigenschaften identische Agrarerzeugnisse und Lebensmittel stets dieselbe Gewinnspanne beim Verkauf an den Verbraucher aufweisen müssen. Ungarn begründete die Regelung mit der Bekämpfung unfairer Handelspraktiken. So würden in Ungarn die herstellenden Unternehmen ihre Marktmacht ausnutzen, um den Einzelhändlern die Gewinnspanne vorzugeben.

Ein solches Verbot der Preisdifferenzierung stellt einen schwerwiegenden Eingriff in die freie Gestaltung der Preise dar. Die Europäische Kommission eröffnete daraufhin ein Vertragsverletzungsverfahren gegen Ungarn, das vorrangig auf einen Verstoß gegen die GMO und subsidiär auf eine Verletzung des freien Warenverkehrs gestützt war. Der EuGH bekräftigte zunächst seinen in der Rechtssache Lietuvos Respublikos Seimo aufgestellten Grundsatz, dass die Aufhebung der Preismechanismen in der EU-Agrarmarktorganisation nicht bedeute, dass nun die Mitgliedstaaten solche Preismechanismen wieder einführen könnten. Denn die GMO wolle „die freie Bestimmung der Verkaufspreise auf der Grundlage des freien Wettbewerbs“. Davon ausgehend stellte der EuGH kurz und bündig einen Verstoß der ungarischen Regelung gegen die GMO fest. Die Frage, welchen Spielraum die GMO den Mitgliedstaaten im Bereich der Bekämpfung unfairer Handelspraktiken gebe, stelle sich in der Rechtssache nicht, da es sich bei der Preisdifferenzierung nicht um eine unfaire Handelspraktik, sondern um ein übliches und notwendiges Instrument des Preiswettbewerbs handele. Zudem sei die Regelung nicht

\footnotetext{
${ }^{31}$ Ibidem, Rn. 37, 56 ff. und 96.
} 
geeignet, das von ihr angestrebte Ziel zu erreichen, wie der EuGH an mehreren Beispielen verdeutlichte ${ }^{32}$.

In seinen Schlussanträgen vom 12.11.2020 hatte auch Generalanwalt Hogan vertreten, dass ein Verstoß gegen die GMO vorlag. Er machte dabei - wie er es ausdrückte - „respektvoll“ deutlich, dass ihn die Verneinung eines Verstoßes gegen Art. 148 GMO in dem Urteil des EuGH in der Rechtssache Lietuvos Respublikos Seimo nicht überzeugte. Stattdessen stimmte er - wie auch vorliegend vertreten - dem umgekehrten Ergebnis von Bobek zu. Selbst wenn dem EuGH folgend eine „Restzuständigkeit“ der Mitgliedstaaten auf dem Feld der Bekämpfung unfairer Handelspraktiken bestünde, liege die ungarische Regelung jedoch nicht im Bereich dieser Restzuständigkeit. Denn anders als die litauische Regelung diene sie nicht der Unterstützung eines fairen Wettbewerbs zwischen Agrarurerzeuger und Abnehmer, sondern schütze die „Marge des Einzelhändlers“ in Ungarn. Faktisch diskriminiere die ungarische Regelung ausländische Marktteilnehmer, da diesen verwehrt würde, durch niedrigere Preise auf dem ungarischen Markt Fuß zu fassen. Dass die Europäische Kommission keine konkrete Vorschrift der GMO benannt habe, gegen die verstoßen worden sei, sah Hogan angesichts des „breiten Anwendungsbereichs“ der ungarischen Regelung als unschädlich an. Die ,freie Bestimmung der Verkaufspreise auf der Grundlage freien Wettbewerbs“ sei „einer der Bestandteile" der $\mathrm{GMO}^{33}$. Mithin wirkte ein ungeschriebenes Prinzip der GMO als Schranke für nationale Preisregelungen.

\section{Zur Frage eines an die Produktionskosten gekoppelten Mindestpreises}

Die vorliegende Untersuchung hat drei rechtsdogmatische Erkenntnisse erbracht. Erstens lässt sich die Zulässigkeit nationaler Preisregelungen im Agrarmarktbereich unabhängig von dem Liberalisierungsprozess nach einem einheitlichen Schema beurteilen. Dieses Schema wird im Wesentlichen aus zwei klar abgrenzbaren Grundkonstellationen gebildet, die an die jeweils geregelten Agrarerzeugnisse und Zielsetzungen anknüpfen. Zweitens hat sich eine EU-primärrechtliche Alternativargumentation ergeben, um das Phänomen der Sperrwirkung des EU-Agrarmarktrechts in Bezug auf nationale Preisregelungen zu erklären. Drittens wurde herausgearbeitet, dass in

${ }^{32}$ EuGH, Urt. v. 11.3.2021, Rs. C-400/19 (Kommission/Ungarn), Rn. 36, 42, 44 und 49 ff.

${ }_{33}$ G. Hogan, Schlussanträge v. 12.11.2020, Rs. C-400/19 (Kommission/Ungarn), Rn. 62, Fn. 24, Rn. 52 f. und 63 ff. 
der Rechtsprechung des EuGH zum EU-Agrarmarktrecht nach der Liberalisierung an dem Grundsatz festgehalten wird, dass die EU im Rahmen ihrer Agrarmarktorganisation weiterhin von einem Verbot nationaler Preisregelungen ausgeht. Dies beruht im Wesentlichen darauf, dass der nunmehr gewollte Wettbewerb auf dem EU-Agrarbinnenmarkt von dem Grundsatz frei verhandelbarer Preise ausgeht und die mitgliedstaatliche Gesetzgebung diesem Grundsatz unterworfen ist.

Die dargestellten Prinzipien lassen sich auch auf zukünftige Fälle anwenden. Als ein Beispiel sei die sowohl in Deutschland als auch auf Ebene der EU teils erhobene Forderung betrachtet, dass der Kaufpreis, den ein Abnehmer von Agrarerzeugnissen einem Agrarurerzeuger zahlt, mindestens die Produktionskosten des Agrarurerzeugers abdecken muss. So hat beispielsweise der deutsche Bundesrat im Februar 2021 die deutsche Bundesregierung anlässlich des Gesetzes zur Umsetzung der Richtlinie (EU) 2019/633 gebeten, „ein allgemeines Verbot des Einkaufs unter typisierten Produktionskosten entlang der gesamten Wertschöpfungskette [...] auf seine praktische Umsetzung“" hin zu prüfen ${ }^{34}$. Die deutsche Bundesregierung äußerte hierzu, dass sie das „Anliegen, die Position der Erzeuger in der Wertschöpfungskette zu stärken“, teile: „Sie wird alle Vorschläge hierzu unter rechtlichen und praktischen Gesichtspunkten prüfen“35.

In ähnlicher Weise hieß es in einem Antrag der Fraktion Bündnis 90/ Die Grünen vom Mai 2021 im Deutschen Bundesrat anlässlich der Beratung des Umsetzungsgesetzes, dass die deutsche Bundesregierung prüfen solle, „,inwieweit sich der Verkauf von Lebensmitteln unter den Erzeugerkosten als unlautere Handelspraktik“ darstelle, um ,im Sinne des Art. 39 Abs. 1 Buchstabe b AEUV zu fairen Preisen" beizutragen ${ }^{36}$. Im Rahmen der Evaluierungsklausel des Umsetzungsgesetzes ${ }^{37}$ - konkret in $\S 59$ Abs. 1 Satz 3 Agrarorganisationen-und-Lieferketten-Gesetz - wurde dann Folgendes aufgenommen: „In die Evaluierung fließen auch die Ergebnisse der Prüfung eines möglichen Verbotes des Einkaufs von Lebensmitteln und Agrarerzeugnissen unterhalb der Produktionskosten ein".

Ein solches Verbot wäre als eine nationale Preisregelung einzustufen, so dass ihre Vereinbarkeit mit der EU-Agrarmarktorganisation zu betrachten ist. Soweit sie verarbeitete Lebensmittel erfasst, die keine Anhang-I-Erzeugnisse darstellen, wird der sachliche Anwendungsbereich der GMO verlassen. Insofern müsste im Hinblick auf die EU-Agrarmarktorganisation lediglich

\footnotetext{
${ }^{34}$ Bundestags-Drucksache 19/26923 v. 24.2.2021, S. 2.

${ }^{35}$ Ibidem, S. 4.

${ }^{36}$ Bundestags-Drucksache 19/29404 v. 5.5.2021, S. 2.

37 Bundesgesetzblatt 2021, Teil I, S. 4063.
} 
geprüft werden, ob deren Funktionsfähigkeit beeinträchtigt wird. Im Übrigen wäre der Maßstab des freien Warenverkehrs anzulegen. Für die folgenden kursorischen Überlegungen sei eine derartige nationale Preisregelung nur im Hinblick auf Anhang-I-Erzeugnisse gewürdigt.

Die Zielsetzung der Regelung ist ein angemessenes Einkommen für die Agrarurerzeuger, so dass es sich um eine Zielsetzung handelt, die auch die GMO verfolgt. Sie wird etwa in Erwägungsgrund 135 der GMO explizit in Bezug genommen: „Gewährleistung eines angemessenen Lebensstandards für die betroffene landwirtschaftliche Bevölkerung". Mithin liegt die erste Grundkonstellation vor, so dass die Regelung nur zulässig ist, wenn die GMO eine entsprechende Ermächtigung für die Mitgliedstaaten enthält. Eine solche Ermächtigung ist jedoch nicht ersichtlich.

Die GMO verfolgt die Zielsetzung mit mehreren ihrer Instrumente. Die Interventionsmechanismen und die Krisenregelungen sollen ebenso wie die Vermarktungsnormen und anerkannten Agrarorganisationen zu einem angemessenen Einkommen der Agrarurerzeuger beitragen. Hinzu kommt die Grundstützung, die das EU-Agrardirektzahlungssystem jedem landwirtschaftlichen Betrieb gewährt. Insofern greift auch hier das Argument, dass der Mitgliedstaat nicht die in der GMO getroffenen Maßnahmen für unzureichend erklären und stattdessen eigene Maßnahmen anwenden darf. So ist es einem Mitgliedstaat beispielsweise ebenfalls nicht möglich, unter Verweis auf ein unzureichendes Einkommen jedem landwirtschaftlichen Betrieb aus nationalen Mitteln eine auf das Doppelte erhöhte Grundstützung zukommen zu lassen.

Einer solch eklatanten Wettbewerbsverzerrung würde unter anderem das Verbot mitgliedstaatlicher Beihilfen entgegenstehen. Gleiches gilt im Agrarmarktbereich. So besagt Ziff. 50 der aktuellen Rahmenregelung der Europäischen Kommission für Beihilfen im Agrar- und Forstsektor vom 1.7.2014, dass nationalen Beihilfen, ,die mit den Vorschriften der gemeinsamen Agrarmarktorganisation unvereinbar sind oder das reibungslose Funktionieren derselben beeinträchtigen würden", nicht genehmigungsfähig sind. Ziff. 67 ergänzt, dass nationale Beihilfen, ,die allein auf der Grundlage von Preis, Menge, Produktionseinheit oder Betriebsmitteleinheit gewährt werden“, geeignet seien, ,ihrer Natur nach auch die Mechanismen der gemeinsamen Marktorganisation" zu stören. Das Verbot des Kaufs von Anhang-I-Erzeugnissen unterhalb der Produktionskosten wäre eine ähnlich eklatante Wettbewerbsverzerrung wie eine direkte Beihilfe. Faktisch würde ein Mindestpreis für alle Anhang-I-Erzeugnisse festgelegt und somit ein Kernelement einer umfassenden nationalen Agrarmarktordnung eingeführt. 
Dies stört das Funktionieren der EU-Agrarmarktorganisation und käme zugleich einer nationalen Preisbeihilfe nahe.

$\mathrm{Zu}$ betrachten ist noch, ob eine solche Preisregelung als unfaire Handelspraktik eingestuft werden könnte und damit dem Anwendungsbereich der Richtlinie (EU) 2019/633, die für den Bereich der Anhang-I-Erzeugnisse als Spezial- und Ergänzungsregelung zur GMO anzusehen ist, unterfiele. Denn der dortige Art. 9 gestattet den Mitgliedstaaten, über die in der Richtlinie (EU) 2019/633 enthaltenen Verbote hinaus „strengere Vorschriften zur Bekämpfung unlauterer Handelspraktiken“ zu erlassen, um ein „höheres Schutzniveau“ sicherzustellen. Allerdings dürfen die „Regeln für das Funktionieren des Binnenmarktes" nicht beeinträchtigt werden. $\mathrm{Zu}$ diesen Regeln gehört auch der durch die GMO ausgestaltete Agrarbinnenmarkt, so dass bei einer Einstufung als Instrument zur Bekämpfung unfairer Handelspraktik die von der GMO verlangte freie Verhandelbarkeit des Preises sichergestellt sein müsste. Hiergegen würde die Preisregelung jedoch verstoßen.

Fraglich ist allerdings bereits, ob der Kauf unter Produktionskosten per se eine unfaire Handelspraktik darstellt. Vielmehr dürfte grundsätzlich - wie bei der Handelsspanne des Einzelhändlers in der Rechtssache Kommission/ Ungarn - ein normales Geschäftsverhalten vorliegen. So gibt es eine Reihe von Gründen, warum es wirtschaftlich sinnvoll ist, ein Produkt für einen zumeist vorübergehenden - Zeitraum unterhalb der Produktionskosten zu verkaufen. Genannt seien nur die Marktentlastung bei einem Überangebot, die Räumung von Lagern für neue Ware, die Einführung neuer Produkte auf dem Markt oder bei einer Mischkalkulation die Verbindung mit dem Verkauf eines Produktes, das einen entsprechend höheren Gewinn abwirft.

Hinzu kommt, dass das Instrument kaum so formbar sein dürfte, dass es geeignet ist, das mit ihm verfolgte Ziel auch zu erreichen. Abgesehen von der ökonomisch unterschiedlich beantworteten Frage, was zu den Produktionskosten zu zählen ist, könnten typisierte Produktionskosten, die einheitlich für ganz Deutschland gelten, die unterschiedlichen Produktionskostenniveaus nicht angemessen abbilden. Dazu sind die regionalen Wirtschaftsverhältnisse - zum Beispiel die Boden- und Pachtpreise, die Energiekosten sowie die Lohnkosten - innerhalb Deutschlands zu divergent.

Würden die typisierten Produktionskosten alternativ an Regionen wie etwa den deutschen Bundesländern ausgerichtet, bliebe das Problem bestehen, da auch die wirtschaftlichen Verhältnisse in den größeren Bundesländern zu heterogen sind. Stattdessen auf die betriebsindividuellen Produktionskosten abzustellen, wäre kaum durchführbar, da dann jeder Betrieb seine Produktionskosten berechnen und nachweisen müsste. Zudem würde als Folge für jeden Betrieb ein unterschiedlicher Mindestpreis gelten, den der Abnehmer 
zu beachten hätte. Hinzu kommt, dass das Produktionskostenniveau einem dauernden Wandel unterliegt, da etwa die Energie- und Futtermittelkosten schwanken, so dass die Produktionskostenberechnung beständig angepasst werden müsste.

Vor allem aber entstünde das grundsätzliche Problem, dass sich die Preise vermutlich auf dem typisierten Produktionskostenniveau zuzüglich einer kleinen Gewinnmarge einpendeln werden. Landwirtschaftliche Betriebe, die unterhalb des typisierten Produktionskostenniveaus erzeugen könnten, würden größere Gewinne erzielen und als Resultat ihre Produktion ausweiten. Neben der dadurch entstehenden Gefahr einer zumindest vorübergehenden Überproduktion träte eine Befeuerung des gesellschaftlich umstrittenen Konzentrationsprozesses in der Landwirtschaft ein, so dass die Zahl der landwirtschaftlichen Betriebe noch schneller abnähme. Tendenziell würde in einem solchen Szenario auch das typisierte Produktionskostenniveau sinken und die über diesem Niveau liegenden landwirtschaftlichen Betriebe zusätzlich unter Druck setzen. Da die Regelung nur auf dem deutschen Hoheitsgebiet Anwendung finden könnte, bedürfte es außerdem einer Außenhandelsschranke gegenüber preisgünstigeren ausländischen Anbietern, die jedoch durch die Regeln des EU-Binnenmarktes und EUAußenhandels verboten ist.

Um die Schwierigkeit zu verdeutlichen, sei der Schweinemarkt gewählt, dessen Preise gemäß dem so genannten Schweinezyklus schwanken. Würden die typisierten Produktionskosten - um einen willkürlichen Betrag zu nehmen - je Schwein bei 120 EUR liegen, dürfte in Deutschland kein Schwein zu weniger als 120 EUR verkauft werden. Liegt der EU-Binnenmarktpreis bei 140 EUR, könnten die deutschen Schweineerzeuger ihre Schweine zu 140 EUR verkaufen. Sinkt der EU-Binnenmarktpreis anschließend auf 100 EUR, würden deutsche und ausländische Abnehmer deutsche Schweine tendenziell nicht mehr kaufen, da deren Preis 20 EUR über dem aktuellen Marktpreis läge. Den deutschen Schweineerzeugern entstünden dadurch erhebliche Mehrkosten, da sie die Schweine entweder als unverkäuflich entsorgen oder weiter halten müssten, wobei eine Haltungsdauer über den üblichen Mastzeitraum hinaus die Qualität des Fleisches negativ beeinträchtigt und dadurch den Wert des Schweines mindert. Steigt der EU-Binnenmarktpreis erneut auf 140 EUR, würden die deutsche Schweineerzeuger versuchen, die weiter gehaltenen Schweine möglichst schnell zu veräußern, um Platz für neue Schweine zu schaffen, und daher einen Preis unterhalb von 140 EUR wählen. Dadurch entstünde ihnen auch in der Preishochphase ein Schaden, da sie ohne den Mindestpreis in der Preistiefphase mit Verlusten und in der Preishochphase mit die Verluste kompensierenden Gewinnen verkauft hätten. 
Hieran zeigt sich, dass eine derartige Mindestpreisregelung nur funktionieren kann, wenn - wird von der Außenschutzproblematik abgesehen - die Regelung EU-weit gilt. Dann würde allerdings für alle Agrarerzeugnisse erneut ein EU-Preissystem entstehen.

Ein Vorschlag im Ausschuss für Landwirtschaft und ländliche Entwicklung des Europäischen Parlaments, Krisenmaßnahmen daran zu knüpfen, dass der Marktpreis unter die durchschnittlichen Produktionskosten fällt ${ }^{38}$, fand im Ausschuss denn auch keine Mehrheit und ging daher nicht in den Bericht des Europäischen Parlaments vom 23.10.2020 zur aktuellen GAP-Reform ein. Stattdessen forderte das Europäische Parlament die Einfügung eines neuen Art. 206a in die GMO, dessen Abs. 1 unter der Überschrift „Weiterverkauf zum Verlustpreis“ lautete: „Landwirtschaftliche Erzeugnisse [...] dürften nicht zum Verlustpreis weiterverkauft werden“39. Hierdurch wird das im deutschen Gesetz gegen Wettbewerbsbeschränkungen geregelte Verbot des Verkaufs unter dem Einstandspreis aufgegriffen. Auch für diesen viel schwächer in den Markt eingreifenden Vorschlag fand sich allerdings auf Seiten des EU-Agrarrates keine Mehrheit, so dass ihn das Europäische Parlament in den Verhandlungen über die GAP-Reform nicht durchsetzen konnte.

Jegliche Preisregelung in Bezug auf Anhang-I-Erzeugnisse berührt den Kernbereich der EU-Agrarmarktorganisation und ist daher politisch und rechtlich besonders sensibel. Der EuGH hat hierzu eine klare Rechtsprechung entwickelt, die eine nationale Preisregelung nur in ganz wenigen Ausnahmefällen zulässt. Während sie im Bereich der EU-Agrarmarktorganisation faktisch nie möglich ist, kann sie über den Weg einer nicht von der EUAgrarmarktorganisation abgedeckten Zielsetzung theoretisch realisierbar sein. Auch in einem solchen Fall gibt die EU-Agrarmarktorganisation jedoch enge Grenzen vor. Da auch auf EU-Ebene das Instrument der Preisregelung prinzipiell nicht mehr genutzt wird, besteht ein Gleichklang zwischen mitgliedstaatlicher Ebene und EU-Ebene. Die Wiedereinführung von Preisregelungen entweder durch direkte Normierung auf EU-Ebene oder durch eine EU-rechtliche Erlaubnis für die Mitgliedstaaten wäre ein agrarpolitischer Richtungswechsel. Dabei dürfte jedoch von der Grundentscheidung für eine EU-Agrarmarktorganisation und damit gegen nationale Marktordnungen nicht abgewichen werden. Eine Revision dieser Grundentscheidung müsste im Wege einer Änderung des EU-Primärrechts erfolgen.

${ }^{38}$ Europäisches Parlament, Ausschuss für Landwirtschaft und ländliche Entwicklung, Berichtsentwurf PE623.922v01-00, Amendments 576-796 v. 12.12.2018, S. 30 f. (Amendment 606).

39 Europäisches Parlament, Ausschuss für Landwirtschaft und ländliche Entwicklung, Dokument P9_TA_PROV(2020)0289 v. 23.10.2020, S. 154. 


\section{BIBLIOGRAPHIE}

Bobek M. (2019), Schlussanträge vom 7.3.2019, Rechtssache C-2/18 (Lietuvos Respublikos Seimo).

Bot Y. (2015), Schlussanträge vom 3.9.2015, Rechtssache C-313/14 (Scotch Whisky Association).

Busse C. (2008), Zu Entstehung und Inhalt der Verordnung (EG) Nr. 1234/2007 über eine gemeinsame Organisation der Agrarmärkte (Einheitliche GMO), „Jahrbuch des Agrarrechts“"Band VIII, Nr 3.

Deutscher Bundestag, Bundestags-Drucksache 19/26923 vom 24.2.2021.

Deutscher Bundestag, Bundestags-Drucksache 19/29404 vom 5.5.2021.

Düsing M., Martinez J. (Hrsg.) (2016), Agrarrecht, München.

EuGH, Urteil vom 23.1.1975, Rechtssache 51/74 (van der Hulst).

EuGH, Urteil vom 13.3.1984, Rechtssache 16/83 (Prantl).

EuGH, Urteil vom 2.7.1987, Rechtssache 188/86 (Lefèvre).

EuGH, Gutachten 2/91 vom 19.3.1993 (IAO).

EuGH, Urteil vom 16.1.2003, Rechtssache C-462/01 (Hammarsten).

EuGH, Urteil vom 9.9.2003, Rechtssache C-137/00 (Milk Marque).

EuGH, Urteil vom 25.3.2004, Rechtssache C-118/02 (Industrias de Deshidratación).

EuGH, Urteil vom 14.10.2004, Rechtssache C-173/02 (Spanien/Kommission).

EuGH, Urteil vom 26.5.2005, Rechtssache C-283/03 (Kuipers).

EuGH, Urteil vom 23.12.2015, Rechtssache C-313/14 (Scotch Whisky Association).

EuGH, Urteil vom 13.11.2019, Rechtssache C-2/18 (Lietuvos Respublikos Seimo).

EuGH, Urt. vom 11.3.2021, Rechtssache C-400/19 (Kommission/Ungarn).

Europäisches Parlament, Ausschuss für Landwirtschaft und ländliche Entwicklung, Berichtsentwurf PE623.922v01-00, Amendments 576-796 vom 12.12.2018.

Europäisches Parlament, Ausschuss für Landwirtschaft und ländliche Entwicklung, Dokument P9_TA_PROV(2020)0289 vom 23.10.2020.

Grabitz E., Hilf M., Nettesheim M. (Hrsg.) (2015), Das Recht der Europäischen Union, München.

Groeben H. von der, Schwarze J., Hatje A. (Hrsg.) (2015), Europäisches Unionsrecht, Baden-Baden.

Hogan G., Schlussanträge vom 12.11.2020, Rechtssache C-400/19 (Kommission/Ungarn).

Kokott J., Schlussanträge vom 16.12.2004, Rechtssache C-283/03 (Kuipers).

Lenz C.-O., Borchardt K.-D. (Hrsg.) (2013), EU-Verträge - Kommentar, Köln 2013. 


\title{
THE ADMISSIBILITY AND LIMITS OF MEMBER STATES' INTERVENTION IN THE FREE DETERMINATION OF THE PRICES OF AGRICULTURAL PRODUCTS. LEGAL DOCTRINAL CONSIDERATIONS IN THE LIGHT OF THE CASE LAW OF THE COURT OF JUSTICE OF THE EUROPEAN UNION
}

\begin{abstract}
Summary
The aim of this article was to answer the question to what extent the Member States may, by adopting national regulations, interfere in the free determination of the prices of agricultural products. Price regulation instruments are an integral part of the Common Agricultural Policy. According to the traditional view, EU secondary legislation on the agricultural market contains an implicit prohibition on national price regulation. Moreover, an alternative justification for such a prohibition may also be derived directly from the TFEU. The article assesses the admissibility of the application of minimum prices for agricultural products and foodstuffs depending on production costs, discussing various aspects.
\end{abstract}

Keywords: agricultural market, price regulation, unfair commercial practices, production costs

\section{AMMISSIBILITÀ E LIMITI DI INTERVENTO DEGLI STATI MEMBRI IN MATERIA DI LIBERA FORMAZIONE DEI PREZZI DI PRODOTTI AGRICOLI. CONSIDERAZIONI DOTTRINALI ALLA LUCE DELLA GIURISPRUDENZA DELLA CORTE DI GIUSTIZIA DELL'UNIONE EUROPEA}

\author{
Riassunto
}

L'articolo si propone di rispondere alla domanda fino a che punto gli Stati membri possano interferire nella libera formazione dei prezzi dei prodotti agricoli attraverso le regolazioni nazionali. Gli strumenti di regolazione dei prezzi sono parte integrante della politica agricola comune. Tradizionalmente, la legislazione derivata dell'UE in materia di mercato agricolo vieta, in maniera implicita, ai Paesi di regolare i prezzi. Una giustificazione alternativa per tale divieto può essere ricavata direttamente dal TFUE. L'articolo valuta l'ammissibilità di applicare i prezzi minimi per i prodotti agricoli e alimentari in funzione dei costi di produzione, sotto vari aspetti.

Parole chiave: mercato agricolo, regolazioni dei prezzi, concorrenza sleale, costi di produzione 